\title{
Advanced Parallelization Strategies Using Hybrid MPI-CUDA Octree DSMC Method for Modeling Flow Through Porous Media
}

\author{
Revathi Jambunathan ${ }^{\mathrm{a}, *}$, Deborah A. Levin ${ }^{\mathrm{a}}$ \\ ${ }^{a}$ Department of Aerospace Engineering, University of Illinois, Urbana-Champaign, IL-61801
}

\begin{abstract}
The advantages of a linear space filling Morton Z-curve to represent a three-dimensional octree structure for the Direct Simulation Monte Carlo method are assessed. The strategies to optimize and exploit the properties of the linearized tree using simple, binary computations are presented. Hybrid MPI-CUDA communications are invoked to facilitate the use of heterogeneous architectures for large-scale computations. Strong scaling studies have shown that the parallelization strategies implemented in this work results in $85 \%$ efficiency, and weak scaling studies show nearly $100 \%$ efficiency for a problem size with 0.34 billion particles and 1.5 million immersed body surface elements. Two types of problems, supersonic external flow over fractal-like immersed body and subsonic internal flow through a porous material are solved using the multi-GPU DSMC solver. The permeability of Morgan carbon felt material is calculated by modeling the diffusion of argon gas through the material and the calculated continuum permeability values match well with published data. Keywords: DSMC, permeability, porous media, MPI-CUDA, large-scale simulations, heterogeneous architectures, forest of linear octrees, Morton-Z curves
\end{abstract}

\section{Introduction}

The impetus towards exascale computing has made available a variety of computational resources and advanced algorithms, allowing the use of particle-based kinetic approaches to understand fluid transport through complex domains. The transport problems include diffusion of electrolytes through porous fuel cells[1, 2, 3], hot combustion gases through soot particulates[4], multiphase flow through porous sandstone materials for oil industry applications[5], as well as diffusion of high-temperature reactive gases through porous Thermal Protection System (TPS) materials[6]. The aerospace industry is especially interested in the TPS materials as they provide a protective skin on space capsules, to shield the vehicle and the crew

\footnotetext{
${ }^{*}$ Corresponding author

Email address: jambuna2@illinois.edu (Revathi Jambunathan)
} 
from aerothermodynamic heating during reentry. High fidelity predictive tools are required to characterize the microstructure of these materials, to gain insights on their degradation process when exposed to the high-temperature environment. The characteristic length of the porous microstructure is on the order of a micrometer, and as a result of the material morphology length scale, kinetic effects become important. The Knudsen number, $K n$, defined as the ratio of local mean free path and the characteristic length of the system, is the usual metric to determine the importance of kinetic effects for a given problem. Direct Simulation Monte Carlo (DSMC)[7] is a well known particle-based kinetic approach which can potentially model such finite- $K n$ rarefied gas flows.

DSMC is a probabilistic method used to solve the Boltzmann equation, which is a complex integrodifferential equation for modeling gas transport problems at all $K n$ numbers. In this method, gas flow is simulated using computational particles, where each particle represents large numbers of real atoms or molecules. Particles are initialized with velocities corresponding to a Maxwell-Boltzmann distribution at a given initial temperature. These particles are then moved to new positions using Newton's equations and appropriate boundary conditions are imposed. Binary collisions are performed between nearest neighbors of particles using a probabilistic approach, and post collision velocities are assigned based on kinetic theory by conserving momentum and energy. In the traditional grid-based DSMC method, the gas particles are mapped to the uniform Cartesian grid cells, and from each cell, particle pairs are randomly selected for a probable collision. To model these inter-particle collisions accurately, a DSMC criteria, requiring the cell size to be less than the local mean free path, is enforced. These DSMC processes, namely, particle movement, mapping particles to cells, and inter-particle collisions are repeated every timestep. If immersed bodies are present, gas-surface interactions are modeled and quantities such as the surface heat flux are computed. Once the flow reaches steady state, number density, average velocity, temperature, and other flowfield macroparameters are averaged over many timesteps, in every cell. The DSMC method has been used for a variety of problems, such as modeling ion thruster plume expansion[8], flow through microdevices (MEMS)[9], predicting surface pressure on Mir space station[10] as well as computation of material permeability[6].

Due to the multi-scale nature of most of the high-speed external and low-speed internal flow problems, regions with higher number density require a more refined grid than the regions with lower number density. To adapt the grid according to the varying local mean free path in such systems, an adaptive mesh refinement strategy has been used in some DSMC methods $[11,12]$ as an improvement over the traditional uniform grid approach. A grid-independent particle pairing strategy for the DSMC method was implemented by Pfeiffer et al.[13] for a two-dimensional Couette flow without immersed bodies. In 2008, a gridless octree DSMC 
method was proposed by Olson et al.[14], to efficiently model gas flows through such multi-scale, highly irregular domains. In this hierarchical tree[15, 16] approach, nearest neighboring particles are grouped into spatial bins or nodes by recursive sub-division, until the region spanned by the node is less than the local mean free path. The nodes that do not undergo sub-division are called leaf nodes and are analogous to the collisional cell of a Cartesian grid. However, Olson et al.[14] have not addressed the issues which arise as a consequence of irregularly-shaped immersed bodies.

Collision frequency, the driving mechanism of gas transport in DSMC, is computed using the number of particles in a cell, collision cross-sections of the particle pair, and volume of the cell or leaf node in a tree. If immersed bodies exist in the domain, some leaf nodes will be cut by the surface of this body, distorting the volume that can be occupied by the gas, and altering the collision frequency. Calculation of the exact volume of these intersected leaf nodes or cut-cells is critical to the fidelity of the method. In our previous work[17], we had extended the octree approach to compute the irregular fluid volume of the intersected leaf nodes and model gas-surface interactions using ray-trace, on a serial DSMC code. Our results have shown that inaccurate volume calculation of intersected leaf nodes, can alter the number density and surface heat flux prediction by $40 \%$ and $15 \%$, respectively. But, even with tree-based methods, DSMC is computationally expensive and requires novel parallelization strategies to exploit hybrid architectures to accelerate its performance.

A general philosophy to improve simulation run-time, is to divide the computational work between distributed memory systems, such as multiple CPUs, and perform DSMC calculations simultaneously on each of the processors. This philosophy is executed using the message passing interface (MPI) protocol in a number of DSMC tools such as SMILE[18], MONACO[19], MGDS[12], SUGAR[11], SPARTA[20] and a 3D gridbased DSMC tool by Wu et al.[21]. Graphical Processor Units (GPUs) are increasingly used as accelerators for high performance computing in many Top 500 supercomputers (June 2016 list[22]), and their ability to accelerate particle methods $[23,24,25]$ is well-known. The Compute Unified Device Architecture (CUDA) technology was developed by NVIDIA in 2007 as a means to launch computations on the GPUs. Wu et al.[23], Gladkov et al.[24], Goldsworthy[26], have demonstrated three to ten times speed-up in their two-dimensional DSMC computations with a single NVIDIA Tesla C2050 GPU, compared to a single CPU processor. But, a single GPU is limited by its memory for real large-scale problems, such as flow over a double wedge[27, 28], or internal flow through irregular structures[17].

The solution, therefore, is to use multiple GPUs, where each GPU is attached to a single CPU, and a hybrid MPI-CUDA paradigm is invoked to transfer particle data from one GPU to another. Such a 
hybrid CPU-GPU parallelization has been employed by Wu et al.[29], and Ivanov et al.[30], to improve the computational efficiency of their 2D and 3D DSMC tools. In terms of computational time, Ivanov et al.[30] showed that a single GPU is equivalent to 10 to $30 \mathrm{CPUs}$, and Wu et al.[29] showed nearly 185 times speedup with 16 GPUs compared to a single CPU. Kashkovsky[31] applied his CUDA-MPI DSMC code to model a 3D reentry capsule, with a nearly 100\% efficiency on 48 GPUs. But, the multi-GPU DSMC tools developed so far have been used to solve smaller problems with simple immersed bodies and have not yet been exploited for large multi-scale problems with linearized octree structures. Most of the tree based adaptive refinement methods involve tree traversal, which is computationally expensive. Even the multi-GPU tree-based particle method presented by Zabelok et al.[25] involved tree-traversal, i.e., pointer chasing, which is time consuming and inefficient [32, 33].

In the present work, we introduce novel algorithms as an efficient alternative to tree-traversal used in pointer-based octree DSMC approaches. We begin by linearizing the octree structure such that only the leaf nodes of the tree are stored and all the interior nodes that undergo sub-division are discarded. We employ a Morton-Z space-filling curve to map the leaf nodes of the three-dimensional octree structure into a one-dimensional array while preserving their locality. Such a Morton Z-ordered data structure enables the identification of the exact location of any leaf node in the tree allowing direct leaf node access, while maintaining the hierarchical nature. In contrast to the widely used pointer-based approach, which results in significant loss of fine-grained parallelism due to non-coalesced memory operations, our algorithms involve simple and fast binary operations, in tune with the acceleration capabilities of the GPU architecture. To optimize the hybrid MPI-CUDA communications involved in the multiple CPU-GPU simulations, we implement asynchronous communication and computations. An important requirement for solvers to perform large-scale simulations is load balanced domain decomposition. Morton-ordered trees have been shown to have significantly improved scalability by Burstedde et al.[34]. In our hybrid approach, we distribute the computational work equally between the CPU processors by dividing the computational domain into subdomains of equal computational load. The CPUs, in turn, transfer the particle data corresponding to their sub-domain to the attached GPU device and all the GPUs concurrently perform the DSMC calculations on the data transferred to them, by employing large number of CUDA threads.

The challenges involved in harnessing the acceleration capabilities of the heterogeneous architectures for large-scale problems required us to develop new algorithms and data structures. These strategies and algorithms are employed in our three-dimensional multi-GPU DSMC solver known as Cuda-based Hybrid Approach for Octree Simulations (CHAOS) and will be discussed in Sec.2. In Sec. 3, we will demonstrate the 
flexibility of the CHAOS DSMC solver by modeling argon gas flow for two types of flow, external flow over a fractal-like aggregate and internal flow through a network of microfibers. The feasability and capability of CHAOS will be demonstrated by computing the material permeability of Morgan carbon felt[35], a candidate TPS material. For the computations discussed in this paper, more than 100 GPUs are used to model gas flow with nearly 0.34 million particles through an irregular material modeled with approximately 1.5 million surface mesh elements. We also present our strong and weak scaling studies which demonstrates the high parallel efficiency of the CHAOS DSMC solver.

\section{Linear Octree Construction, Distribution and Particle Mapping Algorithms}

During the initialization phase of the simulation, the boundary conditions, immersed body properties, and gas species properties are communicated to the GPU device from the CPU host. As the domain is filled with particles, an hierarchical octree structure is constructed on the host CPU. The details of the octree construction and linearization, domain decomposition as well as the algorithms for mapping particles to the linear tree and, asynchronous communications are discussed in the following sub-sections.

\subsection{Octree Construction and Morton Z-order}

An octree is a three-dimensional hierarchical tree[15] data structure, primarily employed to efficiently group nearest neighbor particles into nodes, wherein each node spans a certain volume of the computational domain. In the simplest form, the entire domain is the root which undergoes recursive sub-division to generate the final leaf nodes of the tree. For the DSMC approach, recursive sub-division is performed on the nodes until they satisfy the DSMC criteria, which requires the leaf node size to be less than the local mean free path[7], as was implemented by Olson et al.[14] in their gridless DSMC work. If the computational domain or the problem size is larger than a single CPU processor's memory, the domain is divided into multiple roots and distributed to multiple processors where each processor independently performs recursive division on the root assigned to it, resulting in a forest-of-trees (FOT)[34]. The intermediate nodes of the tree, that are neither the root nor the leaf are called internal nodes. In Figs. 1 and 2, the root and the internal nodes of a two-dimensional quadtree are represented by red circles, and the final leaf nodes are shown in blue along with their global IDs.

The two most-widely used methods to store the tree in processor memory are the pointer-based $[8,25,36]$ and the linearized data structure[32, 33, 37]. In a pointer-based representation, the entire tree, starting from the root to the leaf node (along with the internal nodes) is stored, such that, the parent (red) nodes point 
to the address of the children nodes. As a result of this data structure, when we access a leaf node, either to perform DSMC collisions or compute flow-field macroparameters, the entire tree must be traversed from the root through internal nodes and finally to the leaf using pointers. This type of tree traversal is especially time consuming if the depth of the tree, $L_{\max }$, is high because the computational effort scales as $\mathrm{O}\left(\log L_{\max }\right)$.

In a linearized tree, only the leaf nodes are stored in an array $[32,33,37]$ and all the internal leaf nodes along with the root are discarded. The two most important advantages of the linearized representation compared to the pointer-based data structure are that it requires less memory since only the leaf nodes are stored, and, the data access from memory is computationally efficient due to contiguous storage of the leaf nodes. On the other hand, pointer based approaches may be preferable when grid coarsening is required, since inserting or removing leaf nodes from a linear array would require re-shuffling of data elements in the array. However, even in this case, p4est[34] allows octree coarsening on the fly. In this work, though, the octree is only allowed to sub-divide and any slight over-refinement that occurs relative to the required DSMC criteria is accepted.

\subsubsection{Morton ID and locational key for balanced trees}

In this work, a linearized representation of the tree is employed, such that the leaf nodes are stored according to the Morton[32, 33] order and each leaf node has a unique global ID, called the locational key. The leaf nodes of the linearized octree are distributed to the processors and to efficiently locate the position of the leaf node array, a unique Morton ID is assigned to each leaf node. Morton IDs or locational keys are computed using the method proposed by Frisken and Perry[33]. To demonstrate this method, let us consider a fully balanced two-dimensional quadtree as shown in Fig. 1, with unit dimension in each direction. The locational key is computed using maximum refinement level of the tree, $L_{\text {max }}$, and the position $\left(x_{l o c}, y_{l o c}\right)$ of the left-bottom corner of the leaf. The coordinate of the left-bottom corner of the leaf node N11, shown in Fig. $1(\mathrm{a})$ is $(0.75,0.25)$, and, $L_{\max }=2$ for the quadtree. To compute the locational key or Morton ID of the node $N 11$, we will first convert the $\mathrm{x}$ - and $\mathrm{y}$ - coordinate $\left(x_{l o c}, y_{l o c}\right)$ to binary numbers, $x_{b i t}$ and $y_{b i t}$. The binary numbers are then interleaved and converted to the decimal number system to finally give the locational key of the node. The $x_{\text {bit }}$ is calculated using the following expression, $x_{\text {bit }}=\left[\operatorname{int}\left(x_{l o c} * 2^{L_{\max }}\right)\right]_{2}[33]$, which, for this example, is $x_{b i t}=11$. Similarly, the binary representation of the $y_{b i t}$ for node $N 11$ is equal to 01. Interleaving $[32,33]$ the $\mathrm{x}$ - and $\mathrm{y}$ - binary numbers from left to right and converting to decimal system gives $[1011]_{10}=11$. The value of leaf ID stored in the $11^{\text {th }}$ element of the locational key array (Fig. $1(\mathrm{~b})$ ) is 11, indicating the location of the leaf node in the linear array. This shows that using fast bitwise Morton encoding, leaf nodes can be directly accessed based on the locational key. When the leaf nodes are ordered 
according their Morton ID, they follow a Z-pattern or a Z-order as shown in Fig. 1(a).

\subsubsection{Morton ID and locational key for unbalanced trees}

The Morton order and locational key previously discussed works best for a balanced tree since the value of the locational key is exactly equal to the position of the leaf node in the linear array. However, most real-life problems of interest have gradients in the flow-field and as a result the octree is not balanced, i.e., all leaf nodes will not have the same depth from the root. For example, let us consider an unbalanced quadtree shown in Fig. 2, where leaf nodes 0, 1, and, 6 are at level 1, while leaf nodes 2, 3, 4, and, 5 are at level 2, which is also the $L_{\max }$ of the quadtree. For such unbalanced trees, the value of the locational key is not equal to the position of the leaf node in the linear array. The strategy we have implemented to access the leaf nodes of these unbalanced trees directly is such that, if the leaf node is at a level less than the maximum depth, $L_{\text {max }}$, of the tree, then ghost elements up to the maximum depth are created in the locational key array. At the position of these ghost elements in the locational array, the ID of the physical leaf node is stored. The maximum size of the locational key array is $4^{L_{\max }}$ for the quadtree and $8^{L_{\max }}$ for the octree. For example, the leaf nodes $N 0, N 1$ and $N 6$ (Fig. 2) do not undergo subdivision and ghost elements, shown by dotted blue circles in Fig. 2(b), are created in the locational key array to store the ID of the respective physical leaf node. Note that only the physical leaf nodes, $N 0$ to $N 6$, are stored for flow-field macroparameters, such as number density, velocity, temperature, and the ghost elements are stored only in the locational key array to save memory. Consider node $N 6$ with position $(0.5,0.5)$ in the unbalanced quadtree shown in Fig. 2(a). Using the Morton encoding procedure discussed earlier its $x_{b i t}=10$ and $y_{b i t}=10$, which after interleaving gives the locational key $[1100]_{10}=12$. The leaf ID value in the $i=12$ element of the locational key array is 6 , which is the element we are accessing in the linear leaf node array. This algorithm for evaluating the locational key can be extended to a three-dimensional octree, where based on the $x-, y-$ and $z$ - coordinates of the leaf node and the maximum depth of the octree, the $x_{b i t}, y_{b i t}$ and $z_{b i t}$ are obtained and interleaved as $x y z$ from left to right to obtain the leaf location. This locational key is constructed to prevent tree traversal for unbalanced trees, when particles are mapped to the leaf nodes, which will be discussed in Sec. 2.3 Although, the Morton encoding procedure demonstrated in the above examples is for a root with unit length, it can be easily generalized to roots with any dimension, by non-dimensionalizing the coordinates with the dimension of the root.

The Morton encoding procedure along with the locational key is accurate only when the root of the octree is cubic, i.e., the dimensions of the root node are equal in all directions. If the domain is like a channel, i.e., rectangular (or parallelopiped in three-dimensional problem), such as that shown in Fig. 3, then the domain 


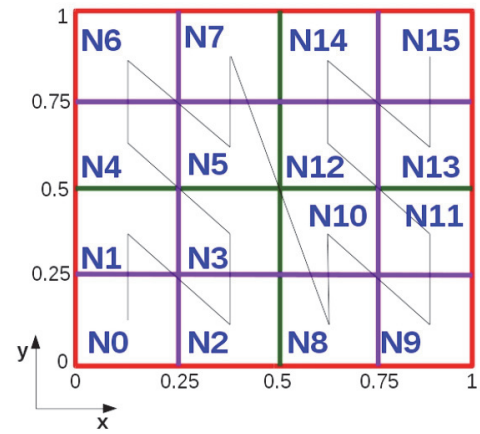

(a) Two-dimensional Domain

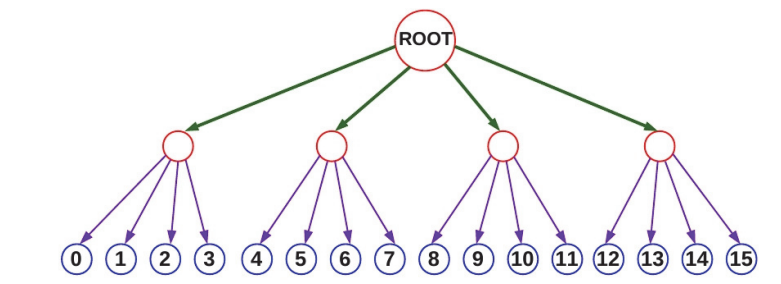

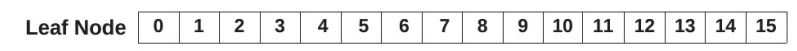

\begin{tabular}{|l|l|l|l|l|l|l|l|l|l|l|l|l|l|l|l|l|} 
Location Key & 0 & 1 & 2 & 3 & 4 & 5 & 6 & 7 & 8 & 9 & 10 & 11 & 12 & 13 & 14 & 15 \\
\hline
\end{tabular}

(b) Linear leaf node array and locational key for fully balanced quadtree.

Figure 1: Balanced quadtree with maximum level of refinement 2.

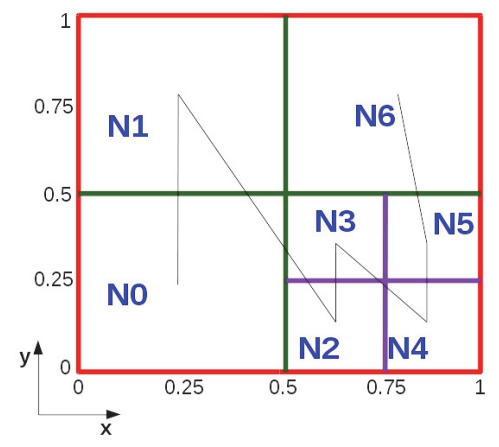

(a) Two-dimensional Domain

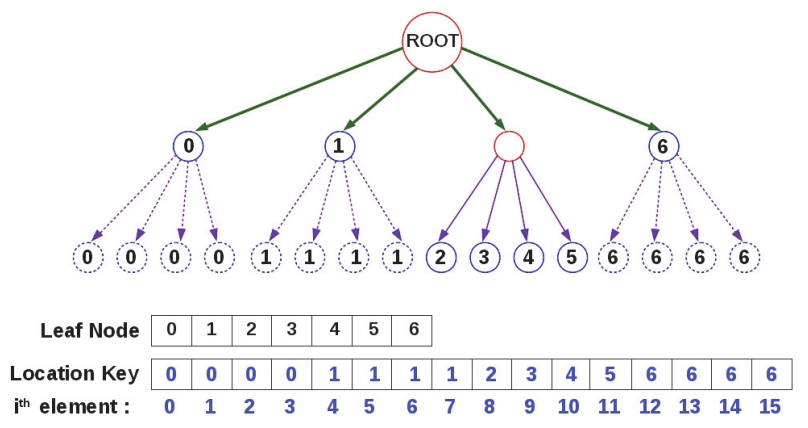

(b) Linear leaf node array and locational key for an unbalanced quadtree.

Figure 2: Unbalanced quadtree with maximum level of refinement 2.

is divided into multiple cubes, where each cubic region forms a root of the forest-of-trees. The leaf nodes of all the trees are sequentially arranged in an array according to the Morton order. For very large-scale problems, a single processor cannot construct an octree structure, grouping all the particles, especially if the number of particles is on the order of billions. To save computational time, the octree construction work is shared by many processors, with each processor containing one or more octree roots. The distribution of the leaf nodes of the forest is discussed next.

\subsection{Domain Decomposition}

The objective of domain decomposition is to equally divide the computational load among multiple processors with the least possible communication cost, in order to reduce simulation run-time. There are many domain decomposition strategies but for DSMC solvers using multiple GPUs, Ivanov et al.[30] and Su et al.[29], decided to divide the domain into sub-domains, and assign one sub-domain to a single GPU. A uniform Cartesian mesh[29, 30] was then constructed on each GPU and the DSMC computations using the 
particles that belonged to that sub-domian were performed independently and concurrently. For adaptive mesh refinement or octree methods, a common strategy $[8]$ is to divide the domain into multiple roots, and distribute one or more roots to each processor. The processor in turn performs DSMC computations on all the leaf nodes belonging to the trees assigned to them. But, for unbalanced trees this strategy does not ensure a balanced load distribution across processors, since some trees have more leaf nodes than others (see Fig. 3, where Root 1 has more leaf nodes than Root 0). An external graph partitioning library such as ParMETIS[38] could be used, however, the overhead required by such libraries to partition the domain is high[34] and may affect scalability for large problems, especially if load balancing is performed dynamically. A simple solution for efficient domain decomposition is provided by the Z-order space filling curve[33, 37], used in the present paper, which allows load distribution to be performed at the leaf level of the forest instead of the root level. As a result, even if the trees are unbalanced and have different number of leaf nodes, the computational load will be divided equally. The Z-order also provides locality, which reduces inter-processor communication.

In our DSMC solver, each processor is assigned a root and based on the procedure described in Sec. 2.1, linearized octrees are independently constructed. Then, each processor determines the total computational weight of the leaf nodes constructed from their root. The computational weight quantifies the amount of run-time required to perform the DSMC calculations in the leaf node and is determined based on the number of particles and the number of immersed body panels in the leaf node. Since the trees are unbalanced and have unequal number of levels and leaf nodes, the leaf nodes are re-distributed to the processors such that all processors have equal computational weight. For example, consider a forest of two linear quadtrees as shown in Figs. 3(a) and 3(b) and let the total number of processors (each attached with a GPU) be equal to 2 , i.e., $N_{p}=2$. In Fig. 3(b), processor 0 constructs a quadtree from root 0 and processor 1 constructs the tree from root 1 . If we assign a weight of one to each leaf node in this example, then the computational weight in processors 0 and 1 is four and ten, respectively. Since the total computational weight of the FOT in this example is 14, each processor must have approximately seven leaf nodes for equal load distribution. Therefore, in the partitioning module, processor 1 transfers the first three leaf nodes, i.e., leaf IDs 4 to 6 , to processor 0, as shown in Fig. 3(b). This load balancing is achieved by cutting across tree 1, which would have been difficult for a pointer-based tree[25] approach. However, for the linearized tree, the array of leaf nodes is simply divided into chunks and distributed to processors, as shown in Fig. 3(b). Finally, the locational key for all the trees are communicated to all the CPU processors using an in-built MPI_allgather function. But, due to memory limitations of the GPU (6 GB for Tesla K20 devices on Bluewaters), only the locational 
key of the trees whose leaf nodes are contained in the GPU's sub-domain is transferred to the GPU (using CUDA API), instead of the keys of the entire forest.

When immersed bodies are present in the domain, one must consider whether they cause significantly more computational load. Initially we assigned intersected leaf nodes a modified weight of 1.5, as compared to the non-intersected leaf nodes with weight of 1 . Our thinking had been that the intersected leaf nodes contain surface triangles of the immersed body, and because gas-surface interactions are time consuming, an additional 50\% computational weight should be provided. However, our scaling studies revealed that this weighting strategy was too simplified. Our analysis, discussed in Sec. 3.3, will show that there are three main factors that contribute to the computational time in DSMC, namely, number of particles, collision frequency, and number of immersed body panels per leaf node. The percentage contribution to the total run-time from each of these factors is obtained by profiling the code for a specific problem for 100 timesteps. From our profiling studies we found that, sampling of macroparameters took negligible time compared to the gas-surface interactions and collision subroutines. Sampling of macroparameters does not require a separate contribution factor, because in effect it depends on the number of particles in the leaf node, the contribution of which we have already accounted. Finally, the total computational weight of a leaf node is computed as a sum of the product of the relative percentages with the value of number of particles, collision frequency and number of immersed body panels. The values of the relative percentage contributions are a user-input and can be modified depending on the problem size. To capture the evolution of the flow physics, the octree structure is destroyed and reconstructed periodically. When a new FOT is created, the previous domain decomposition is deleted and the leaf nodes of the new FOT are, again, equally divided among the processors. This dynamic load balancing ensures scalability for the evolving flow and is achieved at no additional cost or overhead, unlike external graph partitioners.

\subsection{Movement, mapping, gas-surface interactions, sorting and communications}

To perform particle movement in the GPU, a kernel ${ }^{1}$ is launched such that the number of CUDA threads spawned is equal to the number of particles to be moved in the GPU's sub-domain. Each CUDA thread is assigned a particle and all the threads execute the move function independently on their respective particle data. However, when immersed bodies are present in the domain, particle movement is coupled with gassurface interactions (GSI). A code-snippet showing the kernel launch to the GPU and the movement kernel executed by the threads is shown in Appendix 1. Typically, the surface of the immersed bodies are mod-

\footnotetext{
${ }^{1} \mathrm{~A}$ kernel is a subroutine executed in the GPU device using CUDA threads
} 


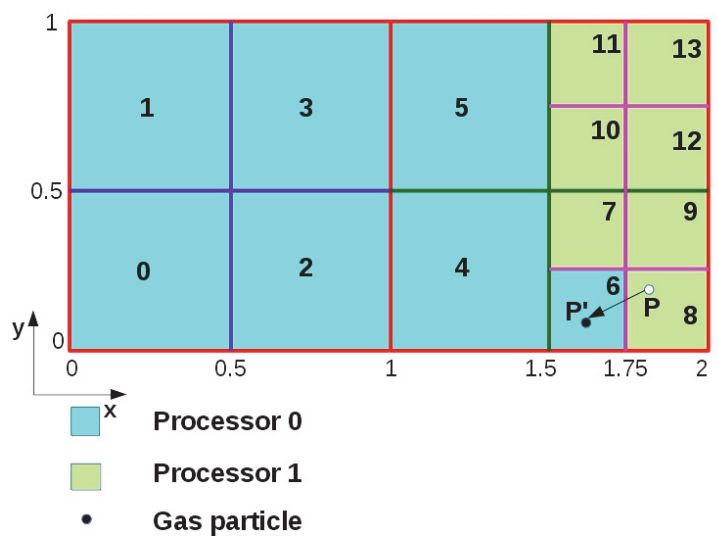

(a) Two-dimensional Domain with two octrees.

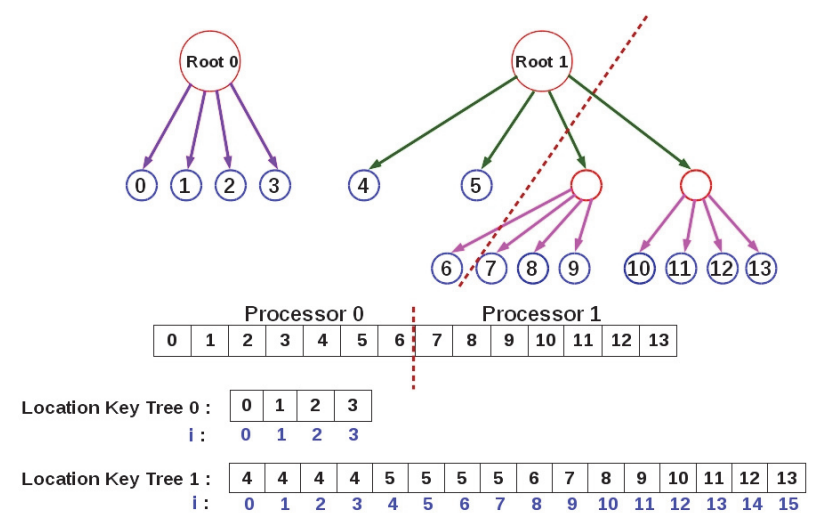

(b) Linear leaf node array and locational key for two quadtrees.

Figure 3: Domain decomposition for a forest of linear octrees.

eled with triangular mesh elements using commercially available software, such as ANSYS ICEM-CFD or MeshMixer. The collision of particles with the surface is detected using the Moller-Trumbore[39] ray-tracing algorithm. A ray along the velocity vector of the particle is cast and checked for intersection with the nearest surface panel. If the time taken by the ray to intersect the nearest panel is less than the timestep, then the particle is reflected from the panel, with post-collision energy based on the accommodation coefficient of the surface. The capability of GPUs to efficiently parallelize the ray-tracing algorithm is well known in the computer graphics community. Therefore, we have exploited this capability of the GPUs for our complex geometries, where the number of surface triangles used to model the immersed body is nearly 1.5 million. In the ray-tracing kernel, each CUDA thread independently determines if the particle assigned to it undergoes a collision with a panel and also computes the time required to impact the surface. The particles that are detected to collide with a panel in a given timestep are flagged as ' 1 ' and others are flagged as ' 0 '. Based on this flagged value, the particle data is sorted using the stable-sort-by-key option in CUDA-Thrust[40]. At a given instant, 32 threads, defined as one CUDA-warp, are launched by the GPU to perform the task allotted to them. Ideally, maximum parallelism is achieved when all the 32 threads of the warp independently perform the same task on different data. If they perform different tasks, then each thread waits until the other threads complete their task which results in loss of parallelism, known as thread divergence[41]. In order to avoid or reduce thread divergence, we have sorted the data so that threads of a warp perform the same task on different particles, i.e. either they undergo reflection with the surface (flag 1) or they are just moved to new positions without surface reflections (flag 0), reducing the possibility of thread divergence. 


\subsubsection{Mapping particles using Morton encoding}

After performing the movement and GSI, some particles may leave the computational domain or the subdomain assigned to the GPU during partition. If the particles leave the computational domain, appropriate boundary conditions are implemented. To generate a list of particles that leave the GPU's sub-domain and move to another sub-domain, particles are first mapped to leaf nodes using their position coordinates in the domain. This is a frequent DSMC occurence, and the advantage of a linearized octree over pointer-based is significant in this mapping procedure. To determine the leaf node, particles are first mapped to the roots of forest using the standard Cartesian grid method, to identify the tree to which they belong. Depending on the maximum refinement level of the tree, the locational key of the particle is generated based on the Morton encoding procedure, discussed in Sec. 2.1. For example, consider a particle $P$ with coordinates $P(1.85,0.2)$ (shown in Fig. 3(a)) which moves to a new location $P^{\prime}(1.6,0.1)$. Based on its new location, the particle is first mapped to Root 1, i.e., it belongs to Tree 1 which has two levels of refinement. The locational key is computed by subtracting the position of the particle with the minimum coordinates of the root, and finally non-dimensionalizing with the size of the root. The $x_{b i t}$ and $y_{b i t}$ in the above example are computed as 10 and 00, respectively. The locational key formed after interleaving the xy bits is 1000, that is 8 in decimal notation. The leaf ID value stored in the $i=8$ position of the locational key for Tree 1 is 6 , and leaf node 6 belongs to processor 0 , as determined during domain decomposition. The algorithm for mapping particle to a three-dimensional octree leaf node is given in Algorithm 1. Particle data is communicated from the GPU of processor 1 to the GPU of processor 0, using the hybrid MPI-CUDA method.

\subsubsection{Particle Sorting and Hybrid MPI-CUDA communications}

After mapping to the leaf nodes, the particles that leave the GPU's sub-domain, like particle $P$, are flagged as 'outgoing particles', and are transferred to the host-CPU using cudaMemcpy. The host-CPUs, in turn, determine the receiving processor ID to which the particle information must be transferred, based on the leaf node partitioning data. The particle data is sent to the respective receiver CPUs using nonblocking point-to-point MPI communication from where it is finally transferred to the GPU co-processor using CUDA. The algorithm for this hybrid MPI-CUDA communication is given in Algorithm $2 . \quad$ The particle data (their position and velocity) is sorted on the GPU, according to their leaf IDs, by employing the stable-sort-by-key option in the CUDA Thrust library (as shown in Algorithm 3 for sorting particle velocity). This allows for storing positions and velocities of particles that belong to the same leaf node in a contiguous manner in the GPU memory and has two important advantages. First, the leaf node is required 
to store only two integers, namely, the number of particles in the node and the starting element ID of the particle in the sorted array. Second, the contiguous storage of particles provides cache coherency, especially later when inter-particle collisions are performed between the nearest neighbors within a leaf node. For example, consider a quadtree constructed with the particle distribution shown in Fig. 4, where particles in the array are sorted based on the leaf node that they belong to. As a result of this contiguous storage, a leaf node, for example, leaf node $N 2$ stores only two integers, the number of particles $=2$ and starting element Id, $i=3$, which means that particles from $i=3$ to $i=3+2$ belong to leaf node $N 2$.

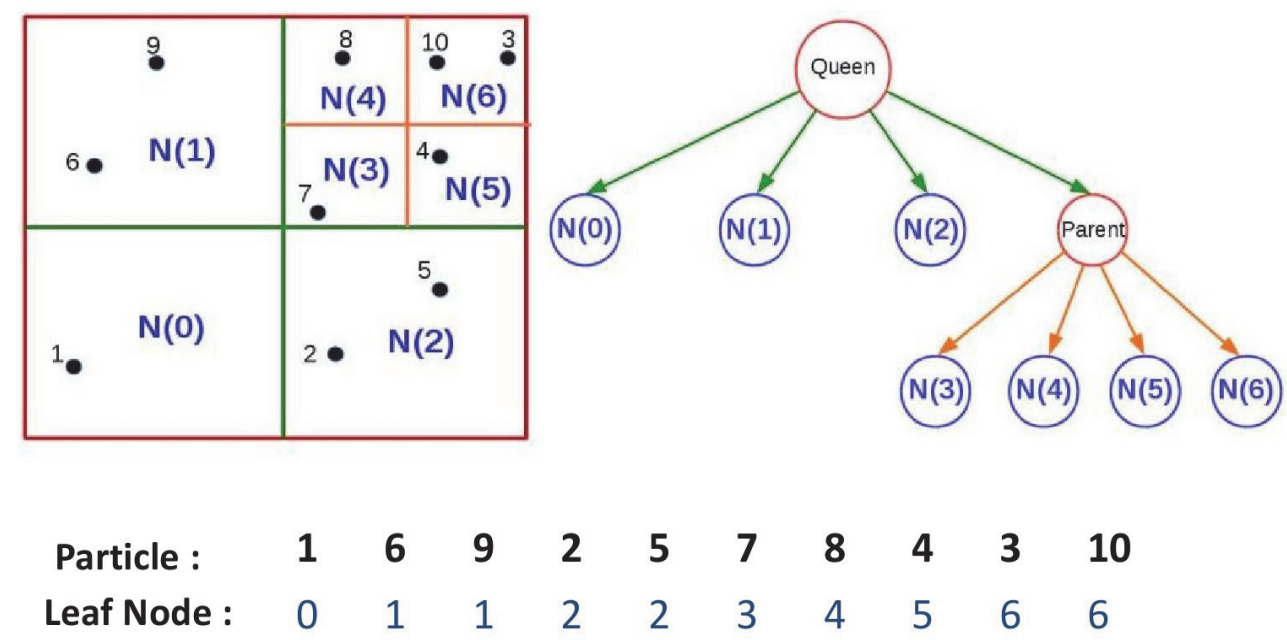

Figure 4: Particle sorting using two-dimensional quadtree structure. The leaf node ID is given in parenthesis. Two arrays, containing the particle and the leaf ID is shown, such that the arrays are sorted by the Leaf ID value.

\subsection{Collisions}

Binary collisions are performed between particle pairs that belong to the same leaf node using the majorant collision frequency scheme[42, 43], which is a function of timestep, number of particles and the leaf node volume. If a leaf node is intersected by an immersed body, the volume occupied by the gas region is irregular and the gas volume is only a fraction of the leaf node volume. This exact volume fraction is computed using a volume of fluid (VOF) method. As described in our previous work[17], we have employed ray-tracing to determine the volume fraction of the leaf node that is occupied by the gas or fluid region. The idea is to divide the intersected leaf node into a uniform Cartesian sub-grid and cast a ray from the geometric centroid of the sub-cells to the corners of the leaf node. Using ray tracing, the closest triangular panel of the surface is detected and the dot product of the ray with the outward normal of this panel is used to detect if the sub-cells lie in either the solid or gas region. In the GPU, the kernel to compute volume is 
launched asynchronously for all the intersected leaf nodes. Each CUDA thread, mapped to a sub-cell of a leaf node, determines if the sub-cell lies in the solid or gas region by ray-tracing over the list of panels in the leaf node. An AtomicAdd operation is performed to add the number of 'fluid' sub-cells and this value is used to determine the fluid fraction. The ratio of gas-phase sub-cells to the total number of sub-cells gives the fraction of volume[44] occupied by the gas. The product of this fraction with the entire volume of the leaf node, provides the final volume of the irregular gas phase. For our simulations, we have used a $(30 \times 30 \times 30)[17]$ uniform sub-grid to compute the volume of fluid fraction in the intersected leaf nodes.

The list of triangles that lie in the intersected leaf node is merged with the octree construction algorithm, to utilize the hierarchical nature of the tree-based approach. Volume computations of intersected leaf nodes are performed only when the octree is constructed, and not at every timestep.

The inter-particle collision kernel is launched by spawning threads equal to the number of leaf nodes in the GPU's domain. Each CUDA thread performs collisions between particles that belong to the leaf node assigned to it. For the GPU implementation, uniformly distributed random numbers are generated by using an in-built CUDA kernel called curand_init to initialize each leaf node with a different initial random seed. A code snippet showing the kernel launch for the generation of unique random number seeds for each leaf node is shown in Appendix 2. A pair of particles from the leaf node is randomly selected for a probable collision and their collision cross-section is computed using the Variable Hard Sphere (VHS)[7] model. If the selected particle pair satisfies the collision probability, then post collision velocities are assigned by conserving momentum and energy.

The flow-field macroparameters, such as velocity, number density, and temperature are sampled in the GPU, after the flow reaches steady state using the last octree structure constructed. Each CUDA thread samples the macroparameters of the leaf node assigned to it. When immersed bodies are present in the domain, heat flux on the panels are computed based on the amount of kinetic energy transferred by the colliding particles to the surface elements.

\section{Results and Discussion}

Our octree DSMC methodology for flow over immersed bodies has been validated in our previous work[17], by comparing the results with a grid based method. In this paper, we have studied the performance of the multi-GPU CHAOS DSMC solver in terms of accuracy, speed-up, and scalability (strong and weak scaling) for two types of immersed bodies, namely a fractal-like spherical aggregate modeled with nearly 1,000 surface triangles and a highly irregular fibrous microstructure, obtained from SEM images of Morgan carbon felt, 
which is a candidate TPS material. All calculations were performed on Bluewaters XK nodes, where each node contains one Tesla K20 GPU employing cudatoolkit7.0. In Sec. 3.1 we present the flow-field results for an external Mach 1.92 argon gas flow over a fractal-like spherical aggregate, for two test cases, with inlet pressures of 0.08 and $1 \mathrm{~atm}$ pressure. In Sec. 3.2, the flow-field results of internal argon gas flow through the porous carbon felt microstructure and permeability studies will be discussed. Finally, scaling studies for both cases for two different number densities will be presented in Sec. 3.3.

\subsection{External flow over fractal structure}

A stream of argon gas particles are introduced at the inlet $(\mathrm{z}=0$ plane $)$ of a $(20 \times 20 \times 20) \mu \mathrm{m}$ domain, with a bulk velocity of $800 \mathrm{~m} / \mathrm{s}$ (Mach 1.92) in the z-direction at a temperature of $500 \mathrm{~K}$. The fractal-like spherical aggregates are suspended at the center of the three-dimensional domain and are assumed to be stationary throughout the simulation. The surface temperature of the fractal structure is held at $300 \mathrm{~K}$. The surface is assumed to be diffusely reflecting, i.e., the gas particles, on collision with the surface, are reflected with an energy equivalent to the surface energy. The DSMC parameters and test case descriptions for the external flow simulations are given in Table 1 . The first three cases have the same inlet pressure $(0.08 \mathrm{~atm})$, but different $\mathrm{F}_{\text {num }}$, number of real atoms represented by a single simulation particle. To test the efficiency of our approach further, Case 4 has an inlet pressure of 1 atm with higher number of simulated particles in the domain. The other boundaries are treated as free boundaries, where particles are allowed to leave the domain if they cross the free boundaries. Particles are introduced with a half Maxwellian distribution at the domain inlet and with a biased full Maxwellian distribution, shifted by the bulk z-velocity component, at the cross-stream boundaries, every timestep. The DSMC parameters for all cases are sufficient to ensure a physical result independent of numerical parameters. The size of the leaf node for the lower pressure cases, namely, Cases 1-3, were nearly one-fourth to one-eighth of the local mean free path, resulting in an over-refinement of leaf nodes in the lower density regions. For Case 4, however, the leaf node size is less than the local mean free path by a maximum factor of one-half. As described in Sec. 2.1, a forest of octrees using Morton Z-curve is constructed for each of the cases, and the leaves of the forest are distributed among the GPUs (Sec. 2.2). The flow-field macroparameters and surface heat flux results for the 0.08 (Case 2) and 1 atm (Case 4) cases are compared below.

The flowfield macroparameters for test Cases 2 and 4, with 0.08 and 1 atm inlet pressure, respectively, are compared along an $X-Z$ plane at $Y=10 \mu \mathrm{m}$ passing through the fractal structure, as shown in Figs. 5 and 6. A detached bow shock upstream of the fractal structure and wake region downstream can be observed from Figs. 5(a) and 5(b). Comparing Mach number contours, it can be seen that the shock structure is 
Table 1: Parameters for external flow over fractal-like spherical aggregates modeled with 2000 triangles.

\begin{tabular}{lcccc}
\hline \hline Case* $^{*}$ & Test Case 1 & Test Case 2 & Test Case 3 & Test Case 4 \\
\hline Number Density $\left(/ \mathrm{m}^{3}\right)$ & $1.3 \times 10^{24}$ & $1.3 \times 10^{24}$ & $1.3 \times 10^{24}$ & $1.5 \times 10^{25}$ \\
Pressure (atm) & 0.08 & 0.08 & 0.08 & 1 \\
FNUM** & 2,000 & 1,000 & 500 & 1,000 \\
Number of simulation particles & $5 \times 10^{6}$ & $11 \times 10^{6}$ & $22 \times 10^{6}$ & $100 \times 10^{6}$ \\
Average number of leaf nodes & 195,935 & 205,917 & 212,315 & $2,123,886$ \\
Time step (s) & $0.4 \times 10^{-9}$ & $0.4 \times 10^{-9}$ & $0.4 \times 10^{-9}$ & $0.2 \times 10^{-9}$ \\
Number of samples & 9,000 & 9,000 & 9,000 & 20,000 \\
Number of timesteps prior to sampling & 2,000 & 2,000 & 2,000 & 8,000 \\
& & & & \\
\hline
\end{tabular}

* Domain size for the above test cases is $(20 \times 20 \times 20) \mu \mathrm{m}$

${ }^{* *}$ The number of real atoms or molecules represented by each simulation particle.

more diffuse for the lower pressure Case 2 as compared to the 1 atm pressure Case 4 . It is also observed that the distribution of normalized number density in the interior region is different for the two different inlet pressures. This is because, Case 4 with higher number density has higher collision frequency and more number of gas-surface interactions, which, in turn, influences the flow-field characteristics in the interior region of the fractal. An increase in the number density, results in smaller local mean free path, as a result of which, the octree leaf nodes undergo more refinement in the higher number density region as compared to the wake region. Further, as a result of higher pressure and higher number density in Case 4, the leaf nodes are more refined in Fig. 5(d) as compared to Fig. 5(c). The spatial variation of pressure normalized by the free-stream pressure for Cases 2 and 4 are shown in Figs. 6(a) and 6(b), respectively. In other words, the thickness of the shock region is smaller for the 1 atm case compared to 0.08 atm case because the shock is stronger in the higher pressure case. Similar observations can also be made by comparing the temperature variation in Figs. 5(b) and 5(a). In both the calculations, the temperature is observed to increase from 500 to $720 \mathrm{~K}$, in the shock region. But, in the interior parts of the fractal structure, the large number of diffuse particle reflections from the surface of the immersed body, held constant at $300 \mathrm{~K}$, lowers the gas temperature to $350 \mathrm{~K}$.

The surface heat flux distribution and three-dimensional velocity streamlines for test Cases 2 and 4 are shown in Figs. 7(a) and 7(b). As the inlet pressure is increased by an order of magnitude, the maximum heat flux at the leading edge of the fractal structure is found to increase by a factor of seven, from 20 to $130 \mathrm{MW} / \mathrm{m}^{2}$. A heat-shielding effect can be observed in the interior region of the fractal structure as well as the region that faces the wake, from the results of both test cases. The velocity streamlines show the trajectory of gas particles through the porous interior region of the fractal structure. For Case 4 with $10^{8}$ particles and 20,000 time-steps, the CHAOS DSMC run-time with 16 GPUs was only 12 hours, demonstrating 
the capability of our approach to model large-scale problems efficiently.

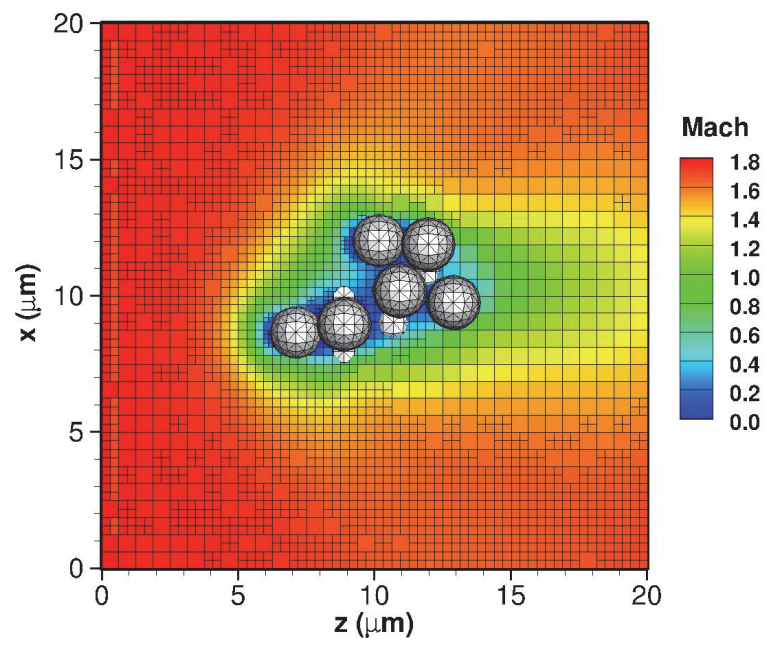

(a) Mach number distribution for test Case 2

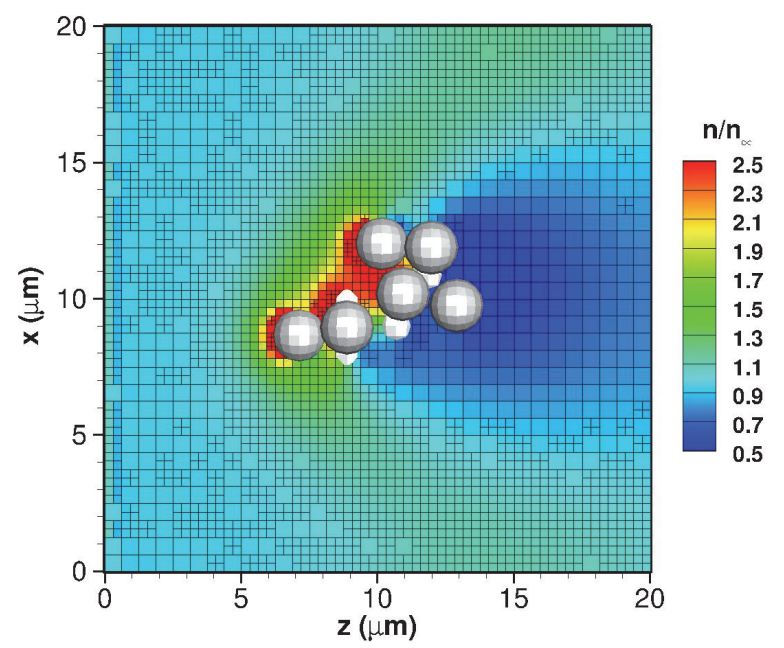

(c) Number density normalized by $n_{\infty}=1.0 \times 10^{24}$ for Case 2 .

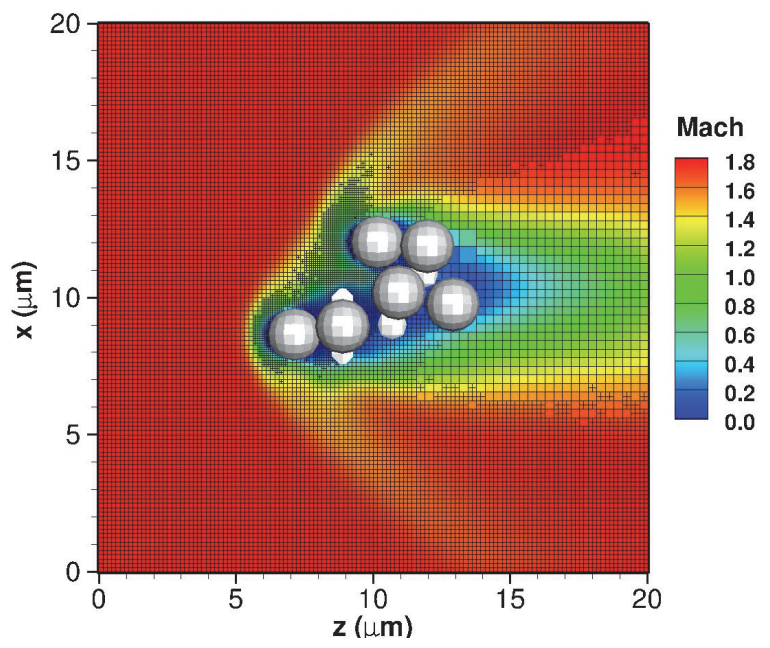

(b) Mach number distribution for test Case 4

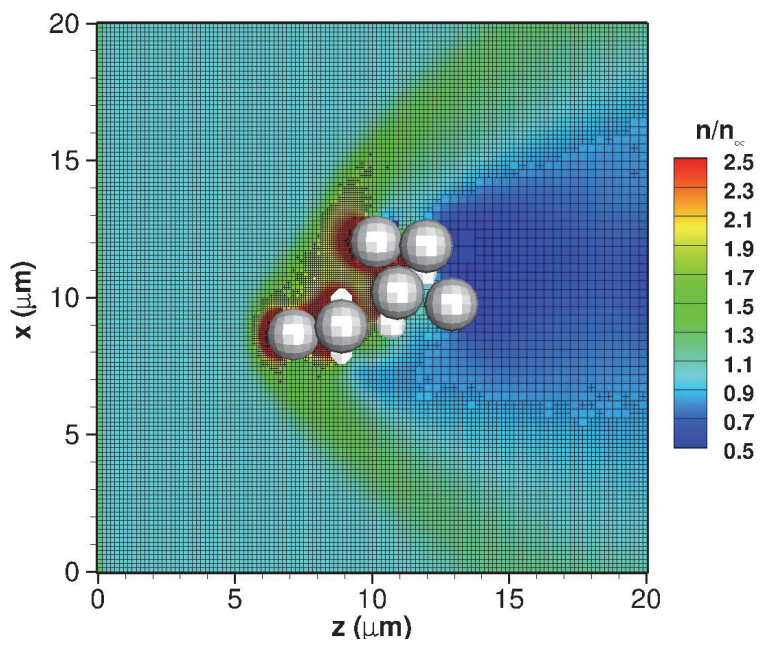

Figure 5: Variation of Mach number and number density along the $X-Z$ plane extracted at $\mathrm{Y}=10 \mu \mathrm{m}$ in the flow-field.

\subsection{Internal Flow through an ablative TPS material}

TPS materials used on the space reentry vehicles are exposed to boundary layer gases with subsonic bulk speed and very high temperatures of up to $2000 \mathrm{~K}$. Permeability is an important material property that aids in our understanding of the diffusion process of these boundary layer gases, which in turn enables the prediction of heat transfer to the material and the resulting degradation. Candidate TPS materials, like Phenolic Impregnated Carbon Ablator (PICA)[45, 46] and in particular Morgan Carbon felt[35], which is a precursor for manufacturing PICA, have a porous microstructure. Due to the small pore diameters of these 


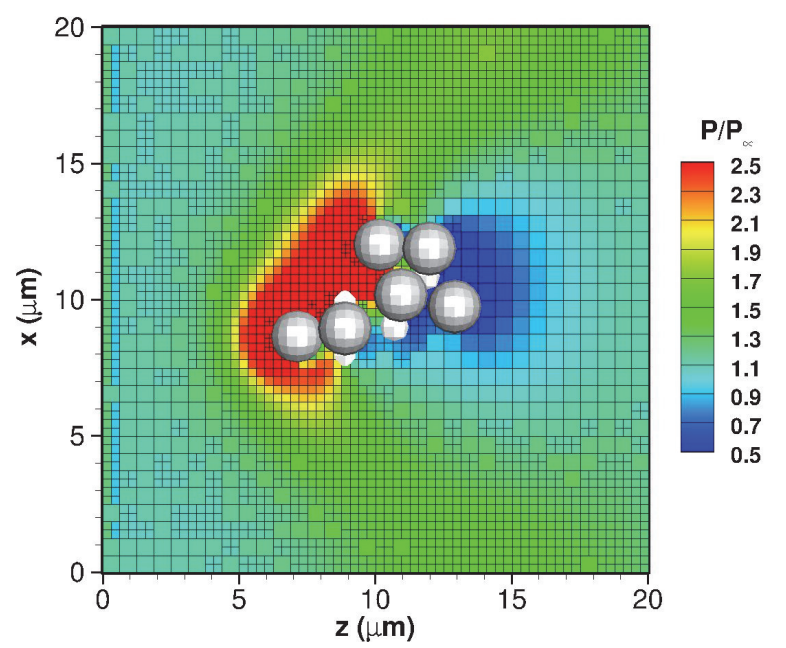

(a) Pressure normalized by $P_{\infty}=0.08$ atm for Case 2

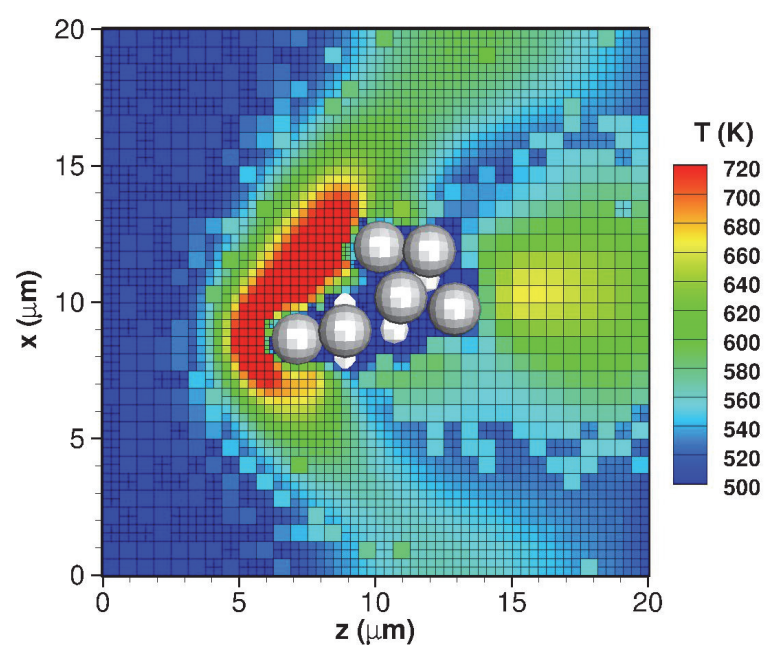

(c) Temperature distribution for test Case 2

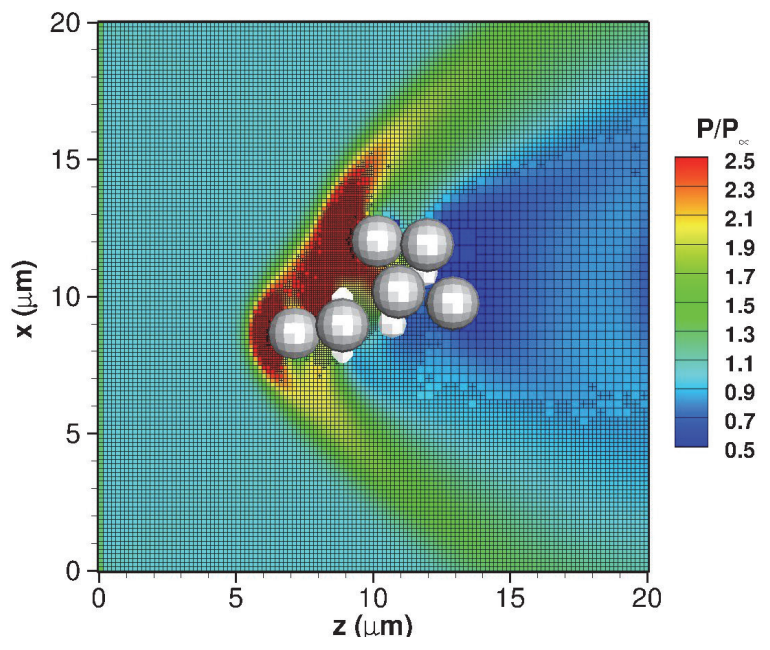

(b) Pressure normalized by $P_{\infty}=1$ atm for Case 4

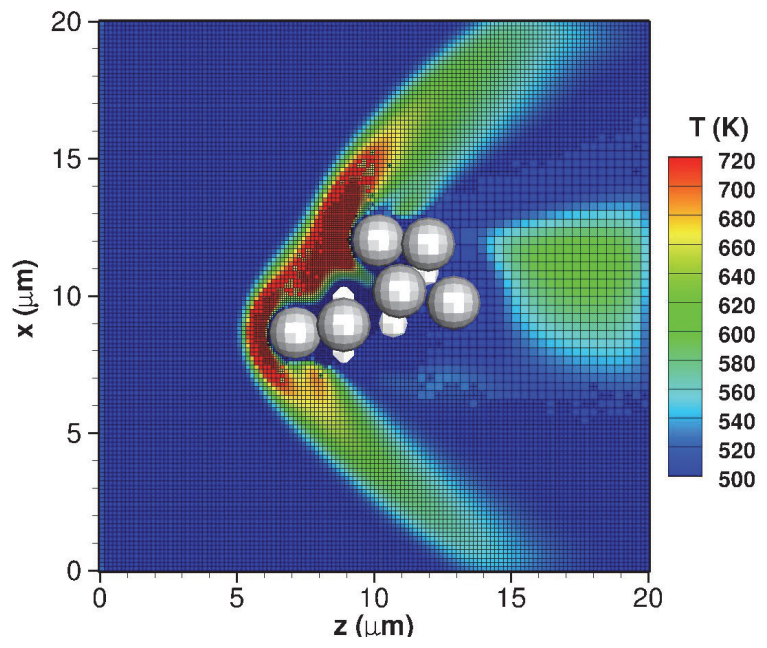

(d) Temperature distribution for test Case 4

Figure 6: Variation of pressure and temperature along the $X-Z$ plane extracted at $\mathrm{Y}=10 \mu \mathrm{m}$ in the flow-field.

material, the flow is rarefied and Darcy's law becomes inadequate to accurately compute the permeability of these fibrous materials. A sample of the Morgan felt material was digitized by Panerai et al.[35], using microtomography and converted to a standard stereolithography file with a surface triangle mesh which is used for all our calculations.

To model the diffusion process, argon gas particles are introduced at the domain inlet with $460 \mathrm{~m} / \mathrm{s}$ initial bulk velocity in the negative z-direction and at a temperature of 2000 K. Since the geometry is irregular, non-periodic, and represents only a small sample of the large scale real material, a pseudo-periodic boundary condition is imposed on the cross-stream boundaries. In this condition, when particles cross the boundary after movement, they are reflected back into the domain specularly, instead of allowing them to leave the 


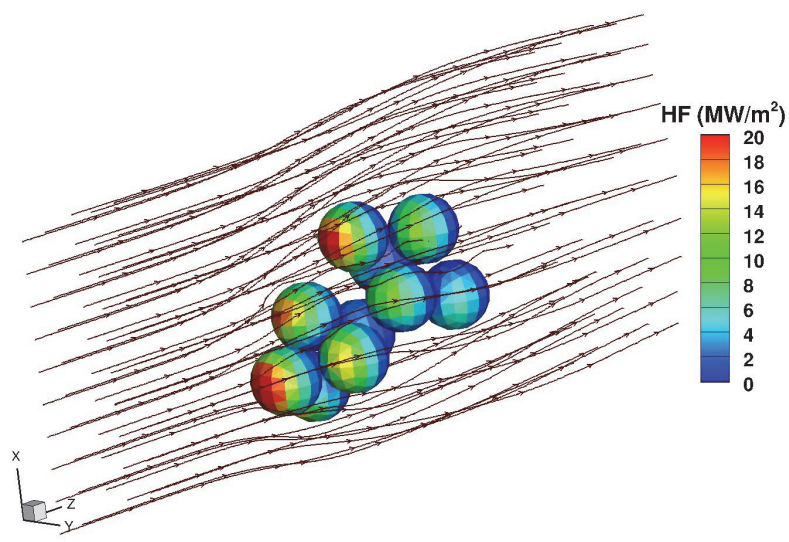

(a) Test Case 2 with $\mathrm{P}=0.08$ atm.

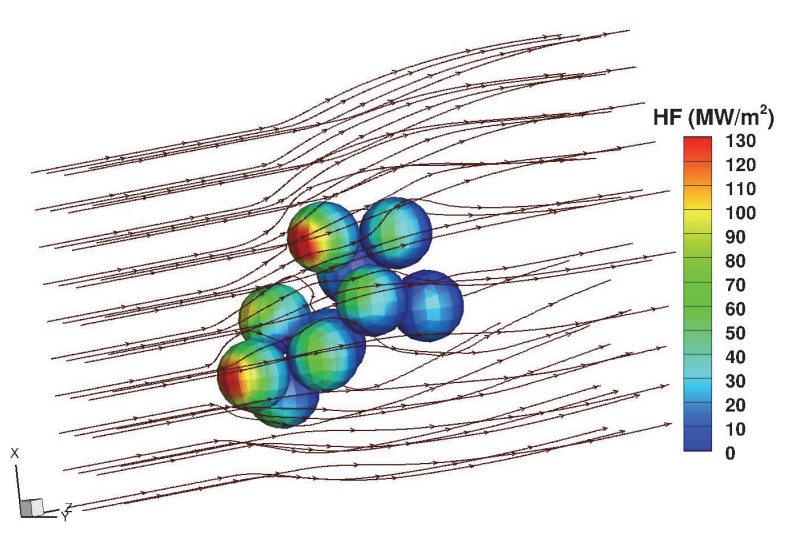

(b) Test Case 4 with $\mathrm{P}=1$ atm

Figure 7: Variation of surface heat flux on the fractal structure, immersed in Mach 1.92 argon gas flow at $500 \mathrm{~K}$, and at 0.08 and $1 \mathrm{~atm}$ and three-dimensional velocity streamlines of the flow.

computational domain. Throughout the calculation, the microstructure is assumed to be unvarying at the constant surface temperature of $300 \mathrm{~K}$. When gas particles collide with the surface of the carbon felt, they are reflected diffusely with energy equivalent to the surface energy. Test cases for flow along the in-plane $(-\mathrm{z})$ and through thickness $(+\mathrm{x})$ direction and their DSMC parameters are given in Tab. 2. Two sets of calculations, defined as test Case A and B in Tab. 2, are performed for bulk velocity in the negative $\mathrm{z}$ (in-plane) direction with inlet number densities of $9 \times 10^{21}$ and $9 \times 10^{22} / \mathrm{m}^{3}$, respectively. To understand the effect of flow orientation on material permeability, two additional sets of calculations are performed with bulk velocity in the $\mathrm{x}$-direction, i.e., the through-thickness direction, (defined as test Case $\mathrm{C}$ and $\mathrm{D}$ in Tab. 2) with the same number densities as for Cases A and B, respectively. The octree structure is created and destroyed every 500 timesteps to capture the evolution of the flow. The computational load is dynamically re-partitioned across the GPUs, until steady state is reached. Macroparameters are sampled on the final octree structure generated at steady state. To test whether the flow has reached steady state, the inlet and outlet mass flow rates were computed in 5 timestep increments. Sampling at steady-state was initiated only when the relative difference in inlet and outlet mass flow rate was less than $0.1 \%$.

\subsubsection{Flow-field and surface heat flux results}

Figure 8 shows the three-dimensional velocity streamlines of argon gas flow through the porous TPS material for Case A and the contour plot of the z-direction velocity $(w)$ in an $X-Z$ plane at $Y=0.202 \mu \mathrm{m}$. From the direction of the streamlines, it can be seen that the bulk velocity is in the negative z-direction (or downward direction). The contour plot of the z-direction velocity shows that the magnitude of the steady- 
Table 2: Parameters for internal flow through a TPS microstructure modeled with 1.5 million triangles.

\begin{tabular}{|c|c|c|c|c|}
\hline Case & Test Case A & Test Case C & Test Case B & Test Case D \\
\hline $\begin{array}{l}\text { Domain size }(\mu \mathrm{m}) \\
\text { Bulk Velocity }(\mathrm{m} / \mathrm{s})\end{array}$ & $\begin{array}{c}404 \times 404 \times 1212 \\
458(-\mathrm{z})\end{array}$ & $\begin{array}{c}1212 \times 404 \times 404 \\
458(+\mathrm{x})\end{array}$ & $\begin{array}{c}404 \times 404 \times 1212 \\
458(-\mathrm{z})\end{array}$ & $\begin{array}{c}1212 \times 404 \times 404 \\
458(+\mathrm{x})\end{array}$ \\
\hline $\begin{array}{l}\text { Inlet Number Density }\left(/ \mathrm{m}^{3}\right) \\
\text { FNUM* } \\
\text { Number of simulation particles } \\
\text { Average number of leaf nodes } \\
\text { Time step (s) } \\
\text { Number of samples } \\
\text { Number of timesteps prior to sam- } \\
\text { pling }\end{array}$ & \multicolumn{2}{|c|}{$\begin{array}{c}9.0 \times 10^{21} \\
4 \times 10^{6} \\
3 \times 10^{6} \\
120,000 \\
2 \times 10^{-9} \\
100,000 \\
100,000\end{array}$} & \multicolumn{2}{|c|}{$\begin{array}{c}9.0 \times 10^{22} \\
4 \times 10^{6} \\
60 \times 10^{6} \\
4,870,160 \\
1 \times 10^{-9} \\
250,000 \\
60,000\end{array}$} \\
\hline
\end{tabular}

*The number of real atoms or molecules represented by each simulation particle..

state velocity decreases from $-160 \mathrm{~m} / \mathrm{s}$ at the inlet to $+20 \mathrm{~m} / \mathrm{s}$ near the fibrous region. This reduction in velocity is due to diffuse reflections from the surface of the material, which causes the reflected gas particles to locally swim against the bulk of the flow. But, due to the large influx of particles introduced at the inlet every timestep, the bulk velocity is still in the negative z-direction, except in the neighborhood of the fibers. Since the material is not uniform, the decrease in bulk velocity is not observed everywhere, but only in the regions where the gas encounters a material obstruction. In the regions upstream of the voids in the microstructure, it can be observed that the bulk velocity is nearly $-60 \mathrm{~m} / \mathrm{s}$ as compared to the regions upstream of the fibers, where the velocity is positive and nearly $20 \mathrm{~m} / \mathrm{s}$. Once the gas flows through the material, the bulk velocity of argon gas increases to $-320 \mathrm{~m} / \mathrm{s}$, since there are no more obstructions encountered, generating a pressure release zone at the outlet. The streamlines, in Fig. 8, show that the gas particles navigate around the fibers through the porous region, demonstrating the capability of the CHAOS solver to model minute details of the flow through the highly irregular network of fibers, at subsonic speed using the DSMC approach. Even without highly complex embedded geometries in the domain, modeling such subsonic flows in any DSMC solver can be challenging due to the long transient time, high collision frequency, and low Mach number.

The effect of increasing the inlet number density by an order of magnitude on the flow-field macroparameters is analyzed by comparing results in the $X-Z$ plane, shown in Fig. 8. Case A and B required 100,000 and 60,000 time-steps, respectively, to obtain the inlet and outlet mass flow rate difference of within $0.1 \%$, i.e., steady state. The steady state mass flow rate of the gas flow in negative z-direction is 53.7 and $537 \mu \mathrm{g} / \mathrm{s}$ for inlet number densities of $9 \times 10^{21}$ and $9 \times 10^{22} / \mathrm{m}^{3}$, respectively. As the number density increases by an order of magnitude, the steady-state mass flow rate also increased by an order of magnitude. Figure 9 shows the variation in the local mean free path, $\lambda$, and z-velocity component of argon gas on the extracted plane for the test cases A and B. A comparison shows that the local mean free path of Case B is lower than that 


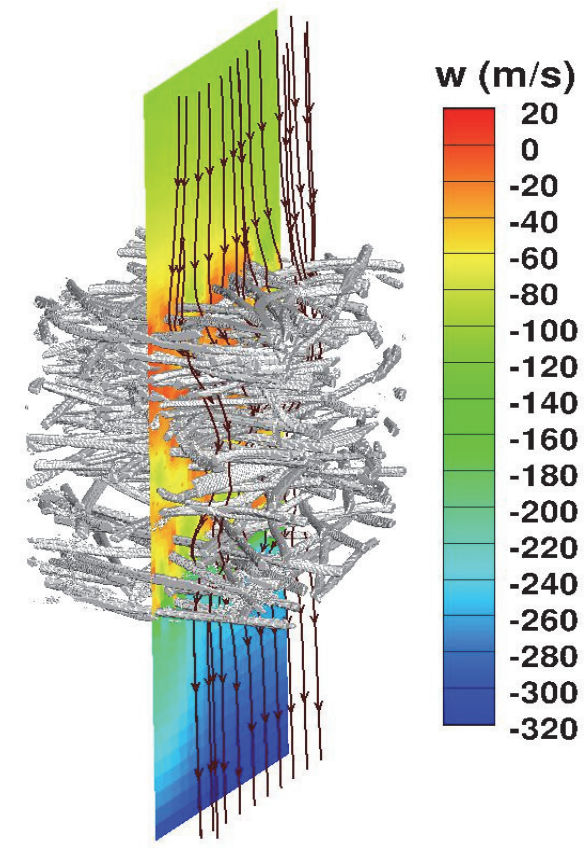

Figure 8: Three-dimensional velocity streamlines of argon gas flow through the TPS microstructure and variation of z-velocity component along a plane extracted at the center of the domain, for Case A (Tab.2)

of Case A, resulting in a more refined octree structure. These local mean free path values are used in the computation of $K n$ number, and it is found that the maximum $K n$ number is 15 and 1.5 and the minimum $\mathrm{Kn}$ is 1 and 0.1 for test Cases $\mathrm{A}$ and $\mathrm{B}$, respectively. These $K n$ number values indicate that Case $\mathrm{B}$ is more continuum-like than Case A, which is consistent with the comparison from number density contour. Figures 9(c) and 9(d) show that for Case B, the decrease in the magnitude of the z-velocity component occurs only very close to the fibers, and has higher negative velocities in the porous region, whereas, for the lower pressure Case A, the velocity magnitude decrease can be observed further upstream of the material, which is typical for a rarefied flow. The normalized number density with respect to the freestream number density, $\mathrm{n}_{\infty}$, for Cases $\mathrm{A}$ and $\mathrm{B}$, and its variation along the extracted plane is compared in Fig. 10. A zoomed-in view of the near-material region is embedded, and the fibers projecting out of this plane are cut for a clearer visual of the contour. As observed for the velocity contour, the increase in number density occurs further upstream for the lower pressure Case A and is more-continuum-like for the higher pressure Case B, which is consistent with the Knudsen number of the flow. The number density increases within the material because the gas particles undergo multiple reflections and become trapped between the porous network of the material. As the gas particles emerge out of the material, their velocity increases and number density decreases, as seen from Figs. 9 and 10, due to a free boundary condition at the exit. 


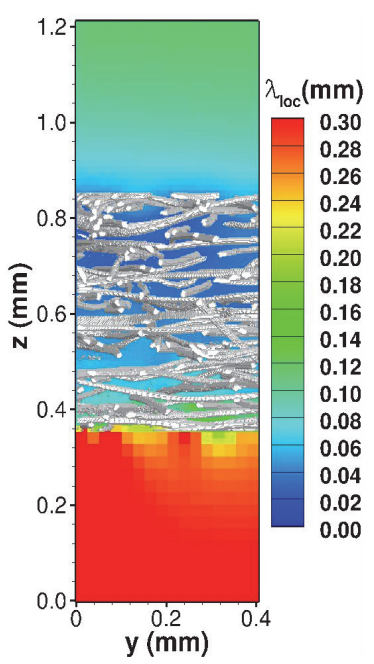

(a) Variation of local mean free path for Case A.

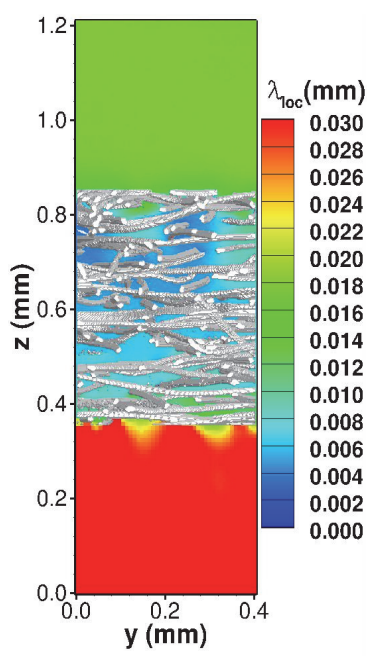

(b) Variation of local mean free path for Case B.

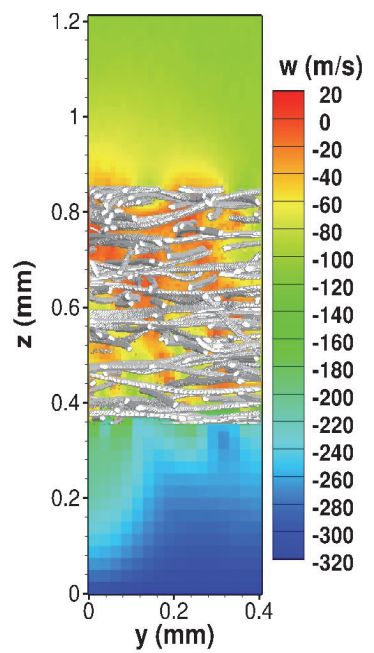

(c) Z-velocity variation for Case A.

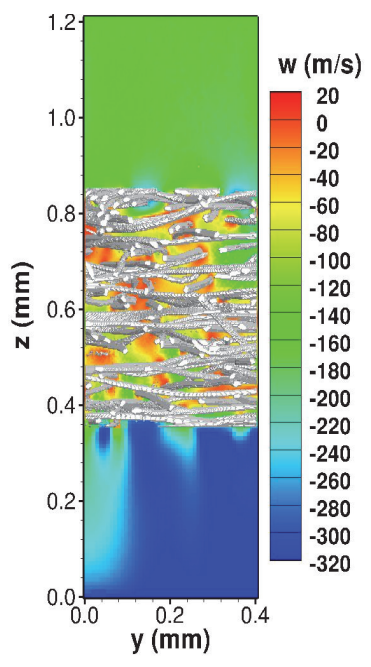

(d) Z-velocity variation for Case B.

Figure 9: The variation of local mean free path (a) and (b), and z-velocity component (c) and (d) on a plane passing through the center of the domain for Cases A and B.

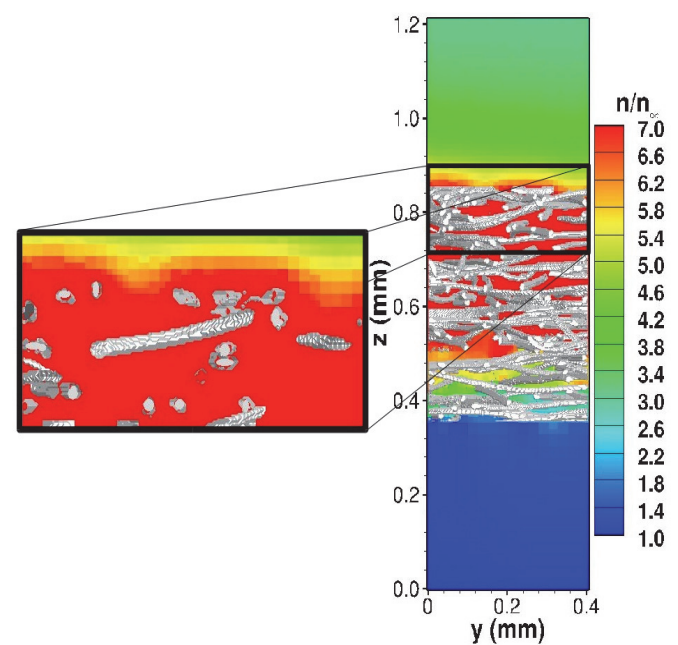

(a) Number density normalized with $n_{\infty}=9 \times 10^{21} / \mathrm{m}^{3}$.

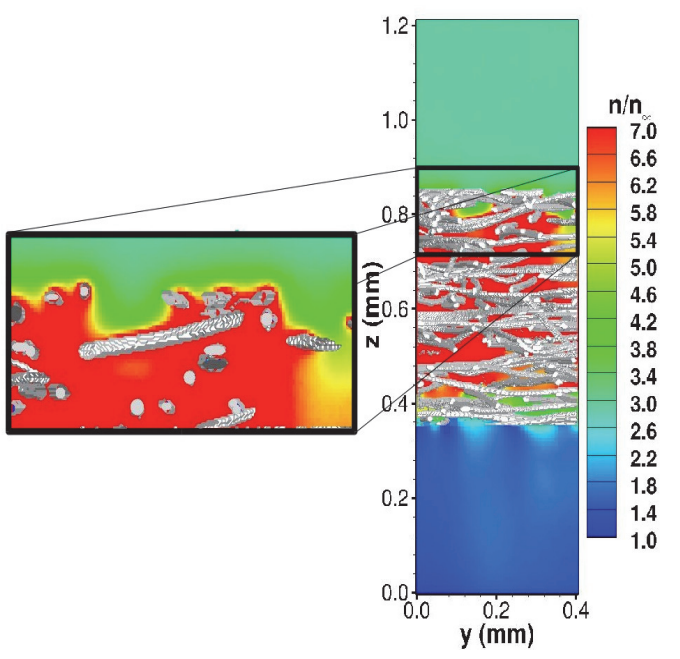

(b) Number density normalized with $n_{\infty}=9 \times 10^{22} / \mathrm{m}^{3}$

Figure 10: Variation of normalized number density in the plane passing through the center of the microstructure for Case A and B.

Temperature variation along the $X-Z$ plane is shown in Figs. 11(a) and 11(b) for test Cases A and B, respectively. The gas temperature decreases from $1600 \mathrm{~K}$ at the inlet to nearly $300 \mathrm{~K}$ near and within the material, because the gas particles undergo fully diffuse reflections, losing energy upon collision, with the surface, which is maintained at $300 \mathrm{~K}$ throughout the simulation. Since the flow domain is not homogeneous in the presence of the fibrous network, the temperature is higher in the porous regions than the near-fiber 
region in the $X-Z$ plane. Figures 11(c) and 11(d) show the pressure contour normalized by the freestream pressure, $P_{\infty}$, for Cases $\mathrm{A}$ and $\mathrm{B}$, respectively. In both the simulations, the ratio of inlet pressure to the free stream pressure is 1.1 and the pressure at the outlet is nearly 0.1 times that of the freestream. But, the pressure contour close to and within the material is more continuum-like for Case B as compared to Case A, similar to the observation made for the number density contour shown in Fig. 10.

The variation of heat flux transferred to the fibrous microstructure and the three-dimensional velocity streamlines of argon gas particles through the material are shown in Figs. 12(a) and 12(b) for Cases A and $\mathrm{B}$, respectively. It can be observed that the heat flux is maximum at the leading edge of the material, which faces the stream of incident argon gas particles. Increasing the inlet number density by a factor of 10 , increased the maximum heat flux by a factor of seven. This increase in maximum surface heat flux is similar to the fractal structure observations for Cases 2 and 4, where the maximum heat flux increased by the same factor.

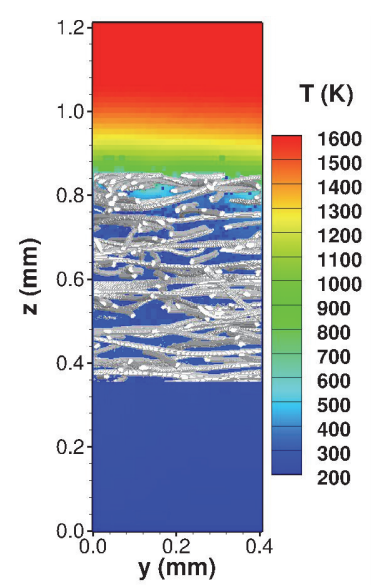

(a) Temperature distribution for Case A.

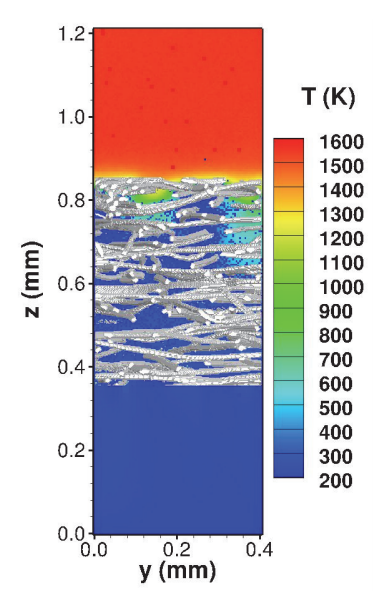

(b) Temperature distribution for Case B.

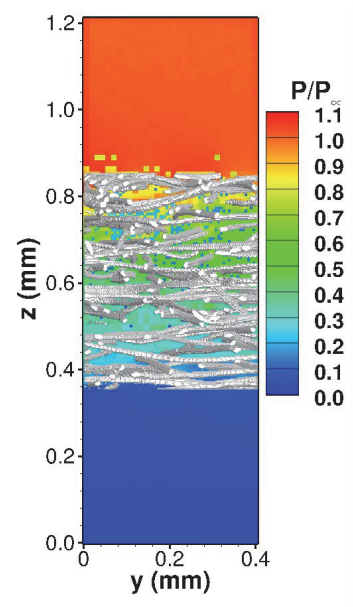

(c) Pressure normal-
ized by inlet pressure
$P_{\infty}=0.011$ atm $($ Case A).

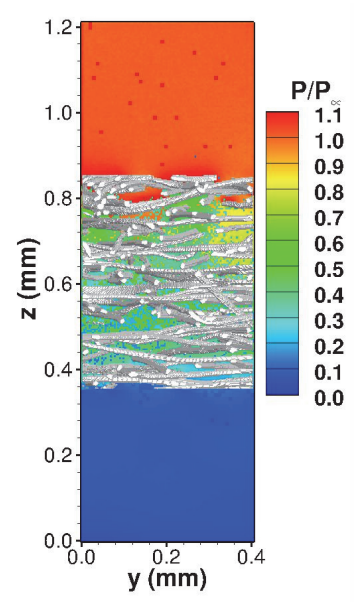

(d) Pressure normalized by inlet pressure $P_{\infty}=0.061 \mathrm{~atm}($ Case B).

Figure 11: Spatial distribution of temperature and pressure in the $X-Z$ plane extracted at the center of the domain, for Cases A and B.

\subsubsection{Material Permeability Calculation}

To enable the comparison of fully kinetic simulation results with experiments[47, 48] of gas transport through porous media, the Klinkenberg formulation[49] is used to estimate the continuum permeability, $K_{o}$, of the TPS material. The effective permeability of the material, $K$, accounting for rarefied slip effects, is 


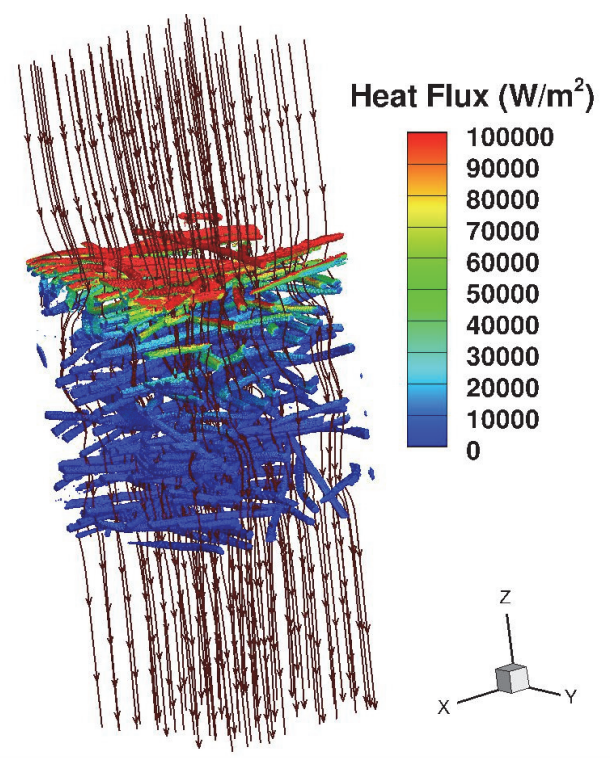

(a) Test Case A.

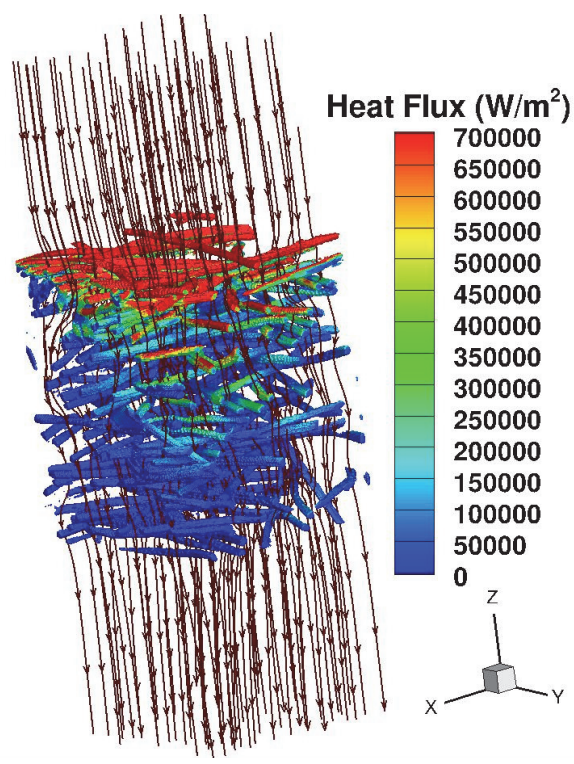

(b) Test Case B.

Figure 12: Spatial distribution of surface heat flux on the microstructure for argon gas flow at average pressure of 0.006 and $0.04 \mathrm{~atm}$.

given as[47],

$$
K=K_{o}\left[1+\left(b / P_{a v}\right)\right],
$$

where, $b$ is the permeability slip parameter that varies with temperature and gas composition, and $P_{a v}$ is the average gas pressure in the domain. $P_{a v}$ is computed by taking an average of the pressure sampled from the DSMC simulations, on the $X-Z$ plane extracted at the center of the domain. From Darcy's law, continuity, and the ideal gas law, the mass flow rate and permeability are related as follows[47],

$$
F=\frac{\mu \dot{m} R T L}{A M \Delta P}=K_{o}\left(P_{a v}+b\right)
$$

where, the quantity $F$ can be used to graphically relate $K_{o}, P_{a v}$, and $b$ with the values of mass flow rate, $\dot{m}$, temperature, $T$, and the pressure difference between the inlet and outlet, $\Delta P$, which are obtained from the DSMC calculations. Since the viscosity co-efficient, $\mu$, molar gas constant, $R$, the molar mass, $M$, of the gas species, the length $L$ and cross-sectional area $A$ of the domain are known, the values of $K_{o}$ and $b$ are obtained from a linear least-square fit of $F$ vs $P_{a v}$ for DSMC calculations performed with different free stream pressures, or $P_{a v}$. The $F$ values for Cases A and B were computed to be $1.014 \times 10^{-6}$ and $1.896 \times 10^{-6} \mathrm{~m}^{2}$, respectively with average pressures of 566.7 and 4,047.7 Pa, respectively. A linear plot of $F$ vs $P_{a v}$ for 
the two calculations resulted in a $K_{o}$ value (slope) of $253 \times 10^{-12} \mathrm{~m}^{2}$ and $b=3,439 \mathrm{~Pa}$ (y-intercept of the line). The effective permeability, $K$, for the low and high pressure rarefied cases, calculated from Eq. 1 , are $1800 \times 10^{-12}$ and $469.2 \times 10^{-12} \mathrm{~m}^{2}$, respectively. Note that the effective permeability values, $K$, are higher than the continuum estimate, $K_{o}$, because of the finite-slip in the rarefied flows, and this slip-effect is more pronounced for the higher Knudsen number Case A.

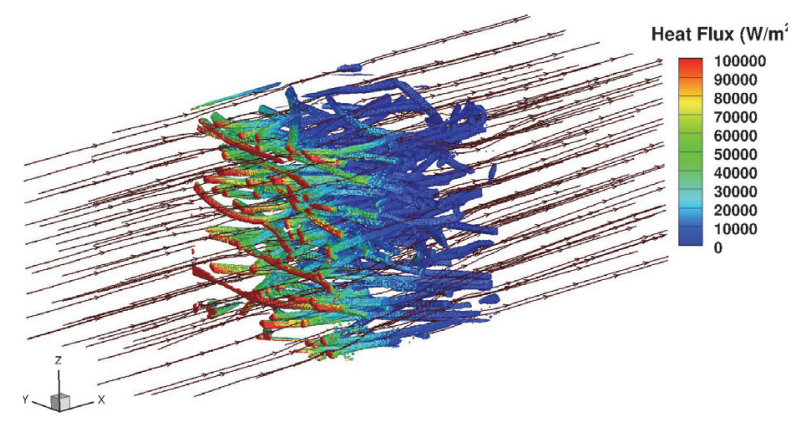

(a) Test Case C.

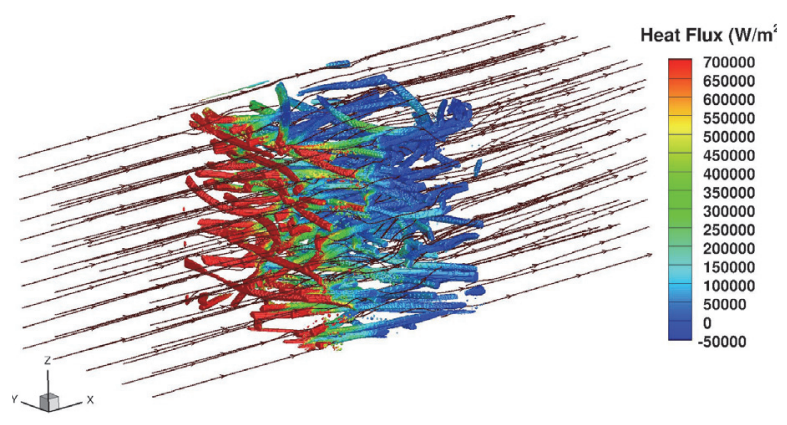

(b) Test Case D.

Figure 13: Spatial distribution of surface heat flux on the microstructure for argon gas flow in $+x$ direction at average pressure of 0.006 and $0.04 \mathrm{~atm}$.

To understand the effect of material orientation on the permeability estimates, we also performed two similar analyses for flow along the through thickness direction, i.e., in the $x$-direction, for the same inlet number densities of Cases A and B, denoted as Cases C and D, respectively. Similar to the $-z$ direction flow, the flowfield macroparameters are analyzed along an $X-Z$ plane passing through the immersed body at the center of the domain. The $F$ values computed for test Cases C and D are $2.058 \times 10^{-6}$ and $2.6 \times 10^{-6} \mathrm{~m}^{2}$, respectively, with average pressures of 394.8 and $3021 \mathrm{~Pa}$, respectively. The linear plot of $F$ vs $P_{a v}$ for the test Cases $\mathrm{C}$ and $\mathrm{D}$ resulted in a $K_{o}$ value of $206 \times 10^{-12} \mathrm{~m}^{2}$ and $\mathrm{b}=9600.3 \mathrm{~Pa}$. Compared to the flow in $-z$ direction, the permeability $K_{o}$ was observed to decrease by $18 \%$ in the through thickness direction, because of more obstruction faced by gas particles for this orientation. The value of the Knudsen correction factor, $b$, was found to increase by a factor of 2.8 by changing the material orientation. That is, when the gas is rarefied, the value of $K=P\left(K_{o}+b\right)$ will increase more for the rarefied flow in $+x$ direction than that for the flow in $-z$ direction. For the same Morgan carbon felt sample, the material permeability, $K_{o}$, computed by Borner et al.[6] was $195 \times 10^{-12} \mathrm{~m}^{2}$ using the SPARTA DSMC solver[20], and $210 \times 10^{-12} \mathrm{~m}^{2}$ using the Geodict software[50]. It can be seen that the material permeability computed using the CHAOS DSMC solver compares well with the published results of Borner et al.[6]. The three-dimensional streamlines and variation of surface heat flux for Cases C and D, shown in Fig. 13, are similar to that of Cases A and B, with a maximum heat flux of 100,000 and $700,000 \mathrm{~W} / \mathrm{m}^{2}$, respectively. 


\subsection{Scaling Studies}

Strong scaling studies were performed on CHAOS by fixing the problem size and increasing the number of GPUs from two to 16, for both, the external flow over fractal-like immersed body (Cases 1 to 4 in Tab. 1), and internal flow through the fibrous microstructure (Cases A and B defined in Tab. 2). All the calculations were performed using Tesla K20 GPUs on the Bluewaters petascale facility and asynchronous communications and computations were implemented to improve the efficiency. For the external Mach 1.92 flow case over spherical aggregates, test Cases 1 to 3 (in Tab. 1) have the same Knudsen number (with $0.08 \mathrm{~atm})$ but different values of $F_{\text {num }}$, or number of simulation particles, while Case 4 (1 atm) has smaller Knudsen number and nearly 100 million simulation particles. The scaling studies for the external flow Cases 1 to 4 were performed for 9,000 time-steps during steady state. The speedup comparison of these cases with the scaling studies of Su et al.[29], is shown in Fig. 14. Su et al.[29] performed similar strong scaling studies using Tesla M2090 GPUs for a two-dimensional supersonic lid-driven flow problem with a uniform Cartesian grid at $K n$ numbers of $0.01,0.005$, and 0.002 .

It can be observed from Fig. 14, that as the problem size increases, from Case 1 to Case 4, the scalability improves, i.e., the speedup line moves closer to the ideal line, consistent with the scaling studies presented by Su et al.[29]. For small problem sizes solved with large number of GPUs, the ratio of communication to computation increases, thereby decreasing the computational efficiency. But, as the problem size increases, the ratio of communication to computation decreases, improving the speedup. CHAOS achieved a speedup of 200 times with 16 GPUs compared to a serial CPU run-time for Case 1 ( $K n=1.3$ and 5 million particles), while Su et al.[29] achieved 85 times speedup for a problem size with $K n=0.01$ and 10 million particles. It can be seen that the speedup of CHAOS compared to a serial CPU is much higher than that published in Ref. [29]. Figure 14 also shows that the scaling for Cases 1 to 4 is more efficient than the scaling results published by Su et al.[29]. One of the main reasons for this, is that Su et al.[29] employed static domain decomposition, which is not optimum especially for supersonic flows with large flow gradients. On the other hand, the dynamic domain decomposition employed in CHAOS accurately captures the gradients during the transient phase and redistributes the computational load to the GPUs, resulting in higher scalability.

It is also observed, in Fig. 14, that Case 4 (1 atm pressure and 100 million particles) showed nearly 100\% scaling efficiency up to eight GPUs, whereas for the 16 GPU simulation, the scaling efficiency decreased to $55 \%$. To understand the reason for this decrease, the normalized distribution of number of particles, leaf nodes, and immersed body panels across the GPUs at steady-state was examined. It can be observed from Fig. 15(a) that the leaf nodes and number of particles were almost equally distributed among the eight 


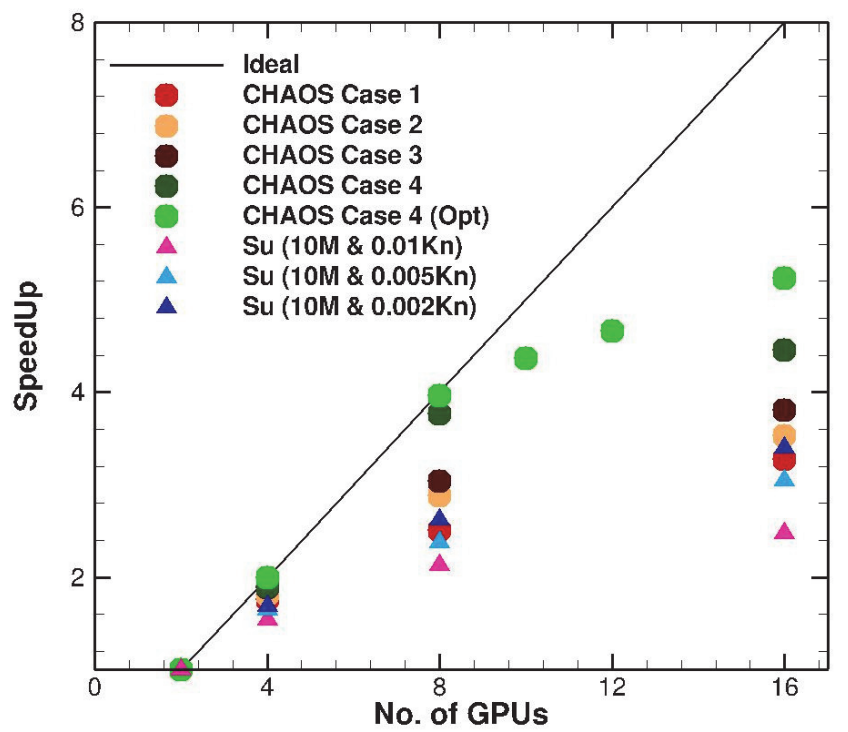

Figure 14: Strong scaling studies for Test Cases 1,2,3, and 4, described in Tab. 1

GPUs, with less than $20 \%$ difference between the minimum and maximum number of particles and leaf nodes per GPU. However, the number of immersed body triangles differed by a large value. The same test case when performed using 16 GPUs (shown in Fig. 15(b)), showed nearly equal distribution of leaf nodes across the GPUs, but the maximum and minimum number of particles in the distribution differed by nearly $80 \%$. For the 16 GPU simulation, it was also observed that the number of particles was low in the GPUs that have a high panel count. As discussed in Sec. 2.2, this led us to conclude that the initial estimate of $50 \%$ additional computational weight for the intersected leaf nodes is not representative of the real computational work, resulting in an imbalance of the actual compute load. That is, this simplistic weighting scheme did not provide good scalability as the computational weights assigned are not related to the actual computational work performed by the leaf node. An improved criteria based on three factors, namely, the number of particles, collision frequency, and number of surface panels of the immersed body, with a weighting of $75 \%$, $20 \%$, and $5 \%$ respectively, for each of the factors were employed. These weights were obtained by profiling the CHAOS solver for 100 timesteps, using the TAU profiler. This new strategy improved the distribution of number of particles across the 16 GPUs as shown in Fig. 16 and increased the scaling efficiency to $65 \%$, as shown in Fig. 14, by the symbol denoted as CHAOS Case $4(O p t)$. It can be observed in Fig. 16, that the number of particles is balanced across the processors, but the speedup (Fig. 14) with 16 GPUs is still not close to ideal. Therefore, we performed additional calculations with 10 and 12 GPUs, and the gradual 
decrease in speedup compared to ideal led us to conclude that the problem size is insufficient to maintain a high ratio of computation to communication.

The speedup for the internal flow through the TPS microstructure used in Cases A (3 million particles), and B (21 million particles), is shown in Fig. 17 and compared with the subsonic lid-driven cavity results from $\mathrm{Su}$ et al[29]. It can be seen that the CHAOS DSMC is more efficient than the subsonic case results published by Su et al.[29], even though the Knudsen number of Case A and B is comparatively higher, i.e., the problem size in terms of to number of inter-particle collisions is smaller. Profiling CHAOS for the fiber cases for a 100 timesteps, during steady state, showed that the weighting factors for number of particles, collision frequency, and number of surface panels for Cases A and B was 50\%,30\% and 20\%, respectively. Compared to the fractal structure cases, it can be seen that the weighting factor for panels needed to be increased from $5 \%$ to $20 \%$ for the fibrous material simulations, because of the very large number of surface triangles that cause an increase in the number of gas-surface collisions. From Fig. 17, it can be seen that the efficiency of CHAOS for Case B with 16 GPUs is nearly 86\%. The problem size of Cases A and B is larger than the external flow Cases 1 to 4, due to large number of immersed body panels. As a result, the speedup plot for the fiber case is closer to the ideal line than the external flow Cases 1 to 4 . The scaling results for Cases A and B were found to be equal, in both, the transient as well as steady phase of the flow. The simulation run-time required to complete 150,000 sampling time-steps for Cases A and B using 16 GPUs was 4.93 hours and 34.5 hours, respectively. The problem size for Case B is nearly an order of magnitude larger than that for Case A, and still, the increase in run-time using the same number of GPUs is less than an order of magnitude, showing that CHAOS is fast for larger problem sizes.

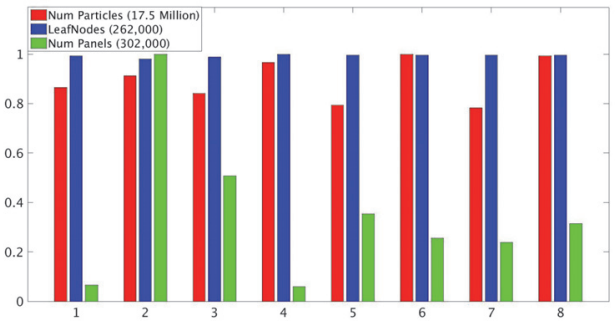

(a) Test Case 4, with 8 GPUs

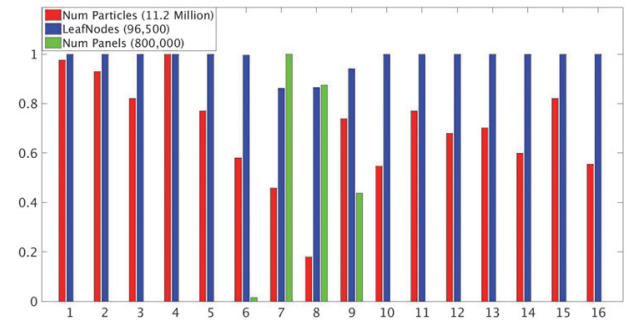

(b) Test Case 4, with 16 GPUs.

Figure 15: Load distribution in terms of number of particles, leaf nodes, and, number of immersed body panels.

Weak scaling studies were also performed for the Morgan carbon felt microstructure[6, 35]. The purpose of weak scaling is to keep the computational load per processor the same resulting in constant run-time with increase in the number of processors and problem-size. Using the eight GPU simulation of test Case B as 


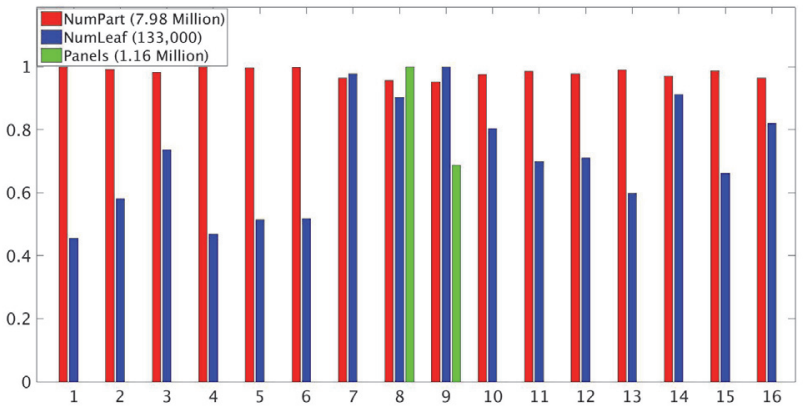

Figure 16: Improved particle distribution across processors with new weighting strategy for the leaf nodes.

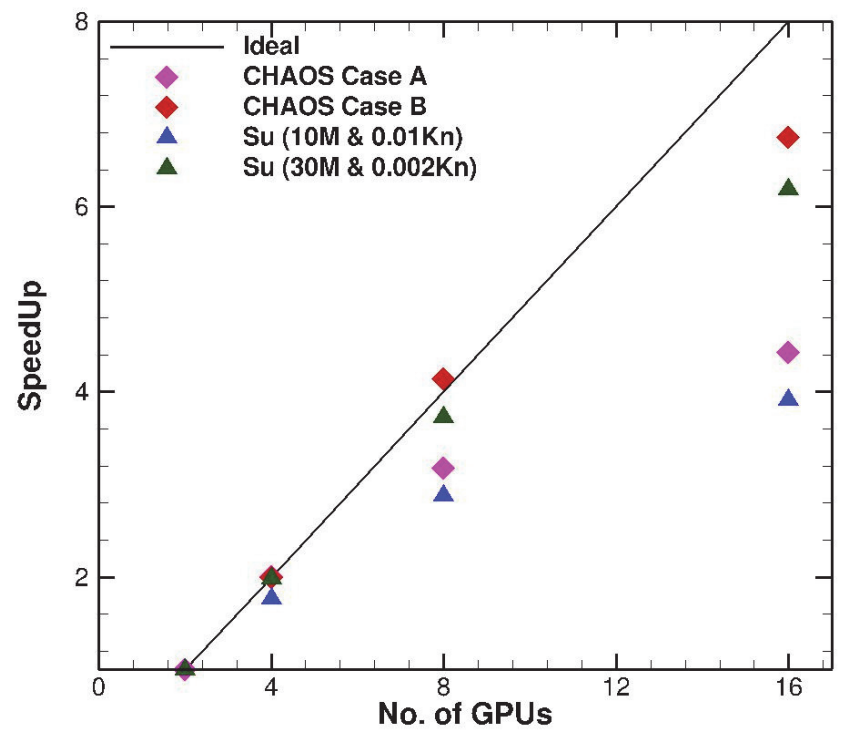

Figure 17: Strong scaling studies for Test Cases A and B, described in Tab. 2

the baseline, we increased the problem size by doubling only the number of simulation particles, and keeping the number density constant, i.e., the total number of leaf nodes was the same. Table 3 gives the DSMC parameters for the weak scaling test cases. Figure18 shows that the scaling efficiency of the CHAOS DSMC is high, suggesting that the algorithms for octree methods that we have developed will be highly scalable. It can be seen that the scaling efficiency is higher than ideal as the problem size and number of GPUs is increased. In conventional DSMC calculations, without complex immersed bodies on the order of 1.5 million triangles, doubling the number of simulation particles results in increasing the compute size by a factor of two. But, in the weak scaling studies that we have performed, we have increased the particle data by a factor of two, while maintaining the same total number of panels. This difference in doubling the number 
Table 3: Parameters for weak scaling studies for flow through carbon felt microstructure.

\begin{tabular}{|c|c|c|c|c|c|}
\hline Number of GPUs & 8 & 16 & 32 & 64 & 128 \\
\hline $\begin{array}{l}\text { Domain size }(\mu \mathrm{m}) \\
\text { Bulk Velocity }(\mathrm{m} / \mathrm{s}) \\
\text { Inlet Number Density }\left(/ \mathrm{m}^{3}\right)\end{array}$ & \multicolumn{5}{|c|}{$\begin{array}{c}404 \times 404 \times 1212 \\
458(-\mathrm{z}) \\
9.0 \times 10^{22}\end{array}$} \\
\hline $\begin{array}{l}\text { FNUM* } \\
\text { Number of simulation particles (mil- } \\
\text { lion) }\end{array}$ & $\begin{array}{c}4 \times 10^{6} \\
21.6\end{array}$ & $\begin{array}{c}2 \times 10^{6} \\
43.56\end{array}$ & $\begin{array}{c}1 \times 10^{6} \\
84.8\end{array}$ & $\begin{array}{c}0.5 \times 10^{6} \\
173.3\end{array}$ & $\begin{array}{c}0.25 \times 10^{6} \\
336.3\end{array}$ \\
\hline
\end{tabular}

*Each simulated particle represents some number of physical particles.

of particles but not total compute size, would alter the total computational work by a small factor. As a result, our weak scaling studies are showing a parallel efficiency of more than $100 \%$, even though the total number of particles per processor is the same.

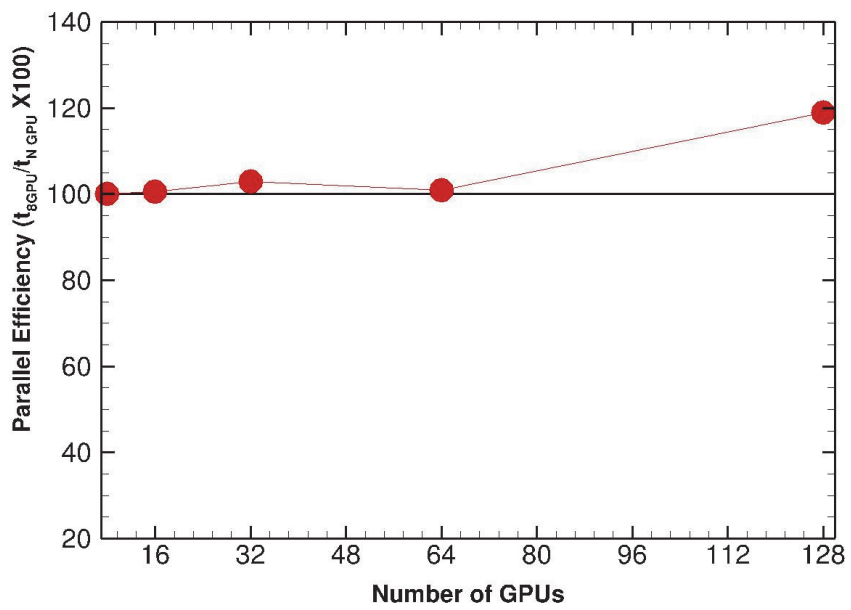

Figure 18: Weak scaling studies for flow through carbon felt with initial number density of $9 \times 10^{22} / \mathrm{m}^{3}$, and equal simulation particles per GPU. (Tab. 3) 


\section{Conclusions}

A hybrid MPI-CUDA parallelization approach, using heterogeneous architectures, for octree DSMC simulations is described. Highly scalable algorithms using fast-bitwise calculations are used to exploit Mortonordered linear octrees for DSMC. Profiling the code and analyzing scaling results showed that there are three factors that contribute to the computational weight of a leaf node or collisional cell in the DSMC method, namely, number of particles, collision frequency and number of immersed body panels. A percentage sum, of all these factors was used to compute the computational weight of a leaf node, which led to equal distribution of computational work to all the GPUs. Our strong scaling results show that such a weighting strategy is key to improving the scalability of the code. For large problems it was shown that CHAOS DSMC is nearly $86 \%$ efficient with 16 GPUs. The results from CHAOS for flow through Morgan carbon felt showed that the permeability of the material in the through-thickness direction compared well with other published permeability data for the material. Weak scaling studies showed that CHAOS is highly scalable even for large problems with 0.34 billion particles and 1.5 millions surface triangles using 128 GPUs, suggesting that CHAOS is a computational tool capable of predicting gas-material macroscopic properties for sufficiently large material sample sizes.

In the future, we will integrate chemical reactions as a module in the solver, to simulate the degradation of the material due to chemical reactions. Further, although the algorithms presented in this paper are for the DSMC method, they can be applied to other kinetic methods, such as the particle-based Particle-InCell $[8,25]$ method that require octrees to model the flow-field. 


\section{Appendix 1}

Code Snippet showing kernel launch to GPU

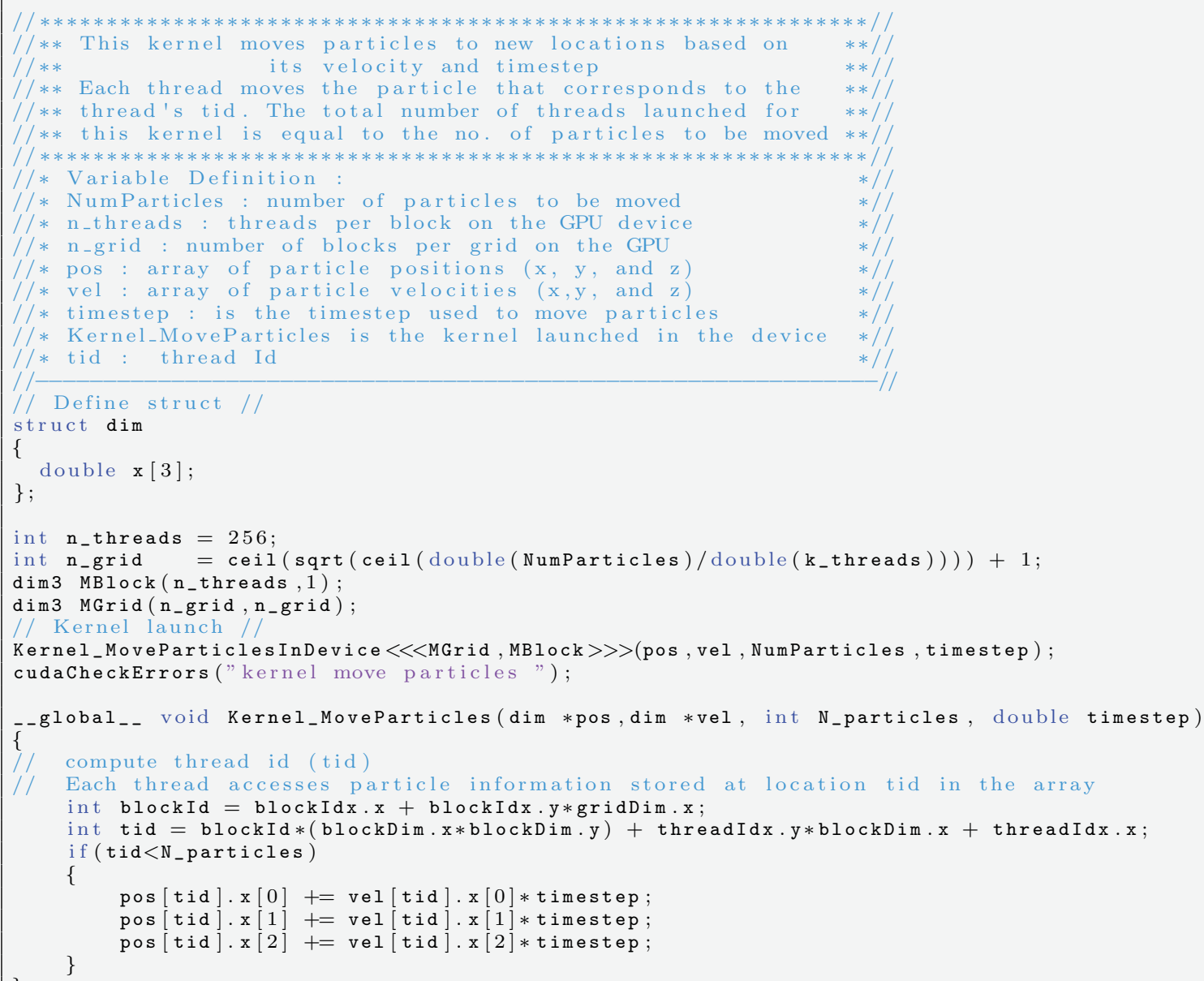




\section{Appendix 2}

Code Snippet showing kernel launch to GPU for unique random number generation

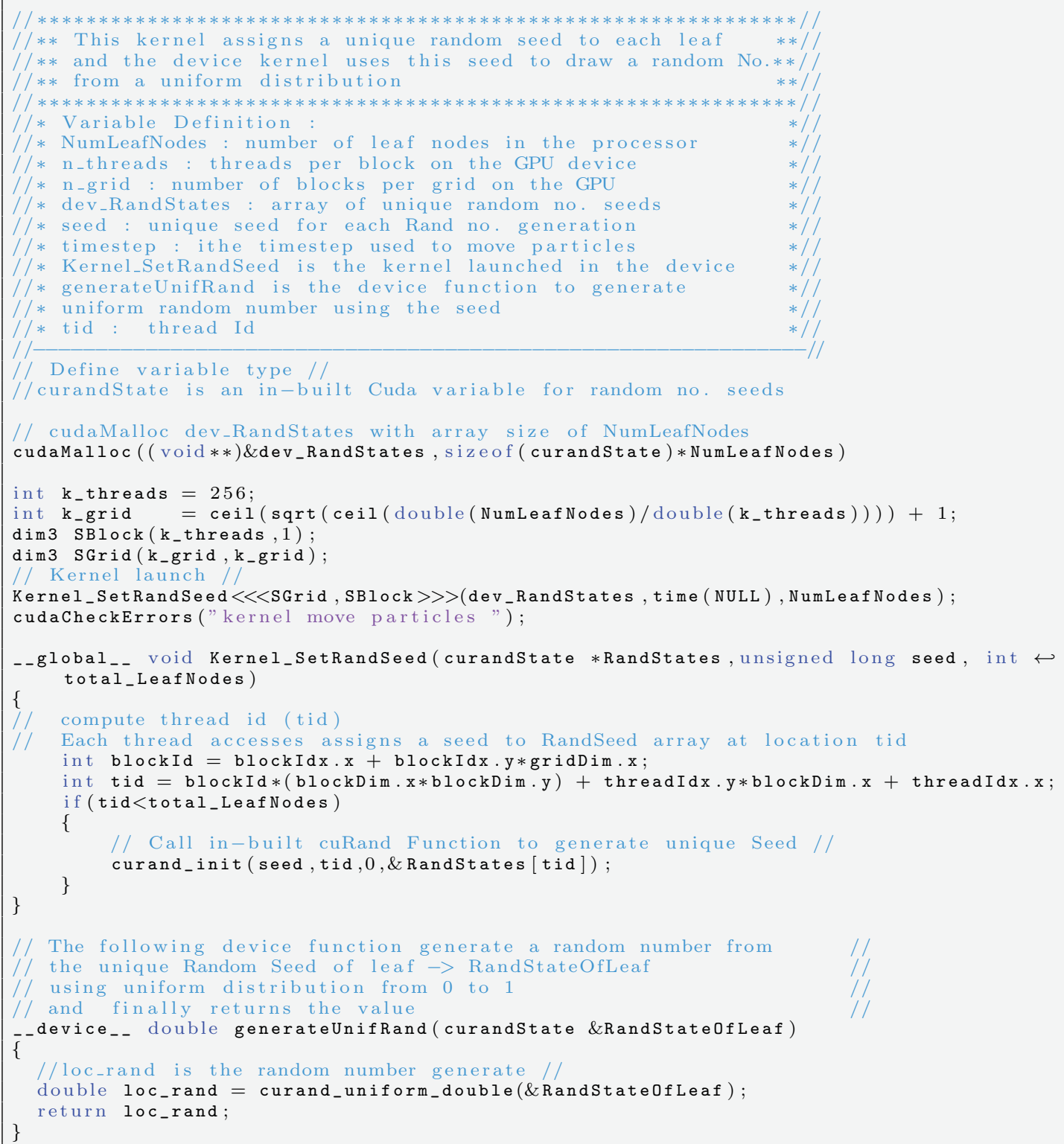



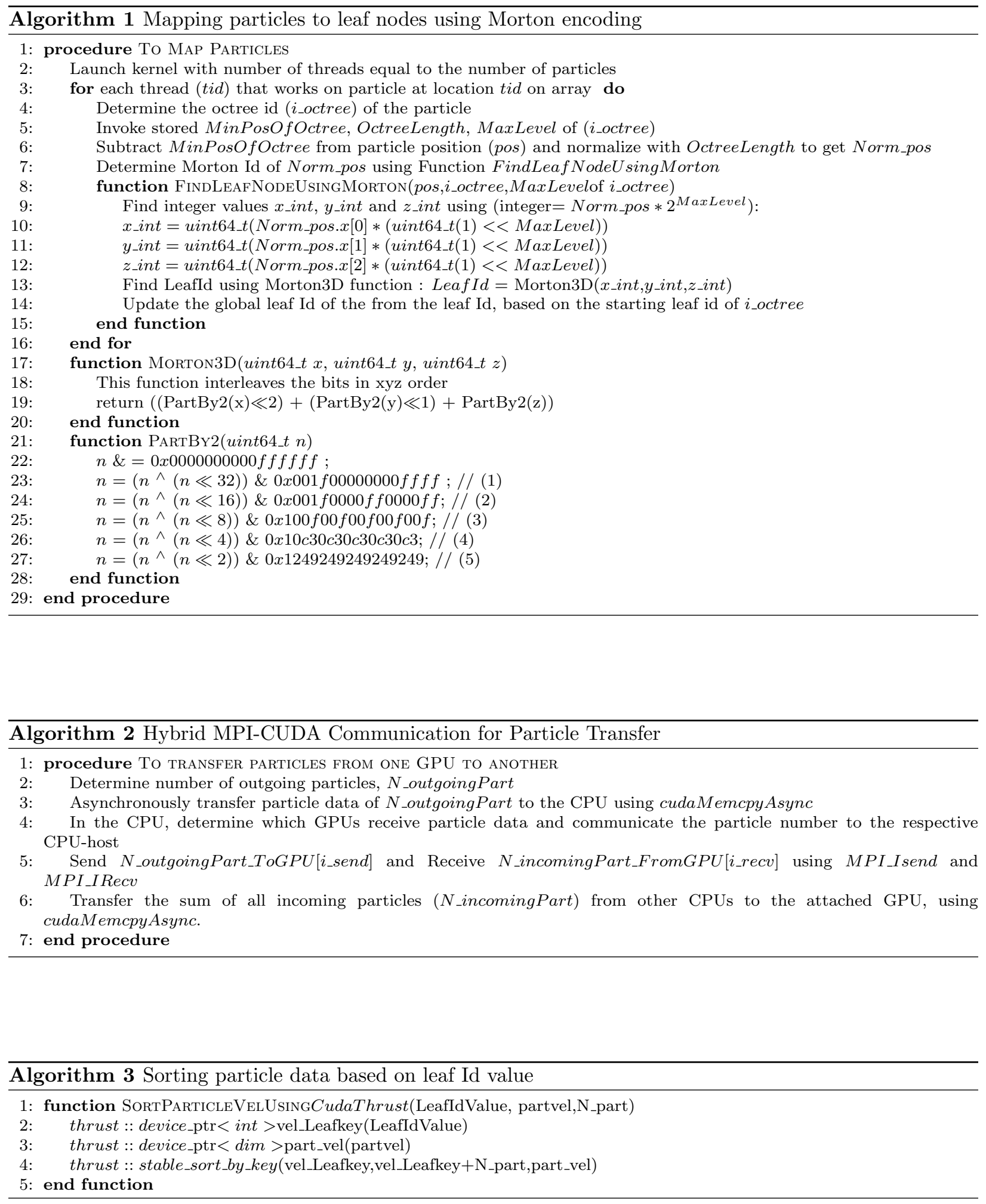


\section{Acknowledgments}

We are grateful for the funding support provided by NASA through the Grant NNX 16AD12G. This research is part of the Blue Waters sustained-petascale computing project, which is supported by the National Science Foundation (awards OCI-0725070 and ACI-1238993) and the state of Illinois. Blue Waters is a joint effort of the University of Illinois at Urbana-Champaign and its National Center for Supercomputing Applications. We are grateful to Professor Wen-Mei Hwu for providing us with access to IMPACT and GEM GPU clusters during the initial stages of code development and for his valuable suggestions. We gratefully acknowledge Dr. Arnaud Borner for the valuable discussions we had and for the carbon felt geometry, as well as Dr. Tong Zhu for the fractal geometry. Contributions on the fractal geometry and the SMILE DSMC results by Tong Zhu at the Pennsylvania State University are greatly appreciated. 


\section{References}

[1] Parikh, N., Allen, J., and Yassar, R., "Microstructure of gas diffusion layers for PEM fuel cells," Fuel Cells, Vol. 12, No. 3, 2012, pp. 382-390.

[2] Cindrella, L., Kannan, A., Lin, J., Saminathan, K., Ho, Y., Lin, C., and Wertz, J., "Gas diffusion layer for proton exchange membrane fuel cells-A review," Journal of Power Sources, Vol. 194, No. 1, 2009, pp. $146-160$.

[3] Mukherjee, P. P., Kang, Q., and Wang, C.-Y., "Pore-scale modeling of two-phase transport in polymer electrolyte fuel cells - progress and perspective," Energy $E$ Environmental Science, Vol. 4, No. 2, 2011, pp. 346-369.

[4] Mueller, M., Blanquart, G., and Pitsch, H., "Modeling the oxidation-induced fragmentation of soot aggregates in laminar flames," Proceedings of the Combustion Institute, Vol. 33, No. 1, 2011, pp. 667674.

[5] Boek, E. S. and Venturoli, M., "Lattice-Boltzmann studies of fluid flow in porous media with realistic rock geometries," Computers 83 Mathematics with Applications, Vol. 59, No. 7, 2010, pp. 2305-2314.

[6] Borner, A., Panerai, F., and Mansour, N. N., "High temperature permeability of fibrous materials using direct simulation Monte Carlo," International Journal of Heat and Mass Transfer, Vol. Accepted for publication, 2016.

[7] Bird, G., Molecular gas dynamics and the direct simulation of gas flows, Oxford Engineering Science Series, Clarendon Press, 1994.

[8] Korkut, B., Li, Z., Levin, D., et al., "3-D Simulation of Ion Thruster Plumes Using Octree Adaptive Mesh Refinement," IEEE Transaction on Plasma Science, Vol. 43, No. 5, 2015, pp. 1706-1721.

[9] Alexeenko, A. A., Levin, D. A., Gimelshein, S. F., Collins, R. J., and Reed, B. D., "Numerical modeling of axisymmetric and three-dimensional flows in microelectromechanical systems nozzles," AIAA Journal, Vol. 40, No. 5, 2002, pp. 897-904.

[10] LeBeau, G. and Lumpkin Iii, F., "Application highlights of the DSMC Analysis Code (DAC) software for simulating rarefied flows," Computer Methods in Applied Mechanics and Engineering, Vol. 191, No. 6, 2001, pp. 595-609. 
[11] Korkut, B., Wang, P., Li, Z., and Levin, D. A., "Three Dimensional Simulation of Ion Thruster Plumes with AMR and Parallelization Strategies," 49th AIAA Joint Propulsion Conference, 2013.

[12] Nompelis, I. and Schwartzentruber, T. E., "Strategies for Parallelization of the DSMC Method," AIAA Aerospace Sciences Meeting Texas, Vol. 2000, 2013, pp. 55455.

[13] Pfeiffer, M., Mirza, A., and Fasoulas, S., "A grid-independent particle pairing strategy for DSMC," Journal of Computational Physics, Vol. 246, 2013, pp. 28-36.

[14] Olson, S. E. and Christlieb, A. J., "Gridless DSMC," Journal of Computational Physics, Vol. 227, No. 17, 2008, pp. 8035-8064.

[15] Barnes, J. and Hut, P., "A hierarchical O (N $\log \mathrm{N}$ ) force-calculation algorithm," Nature Publishing Group, 1986.

[16] Hernquist, L. and Katz, N., "TREESPH-A unification of SPH with the hierarchical tree method," The Astrophysical Journal Supplement Series, Vol. 70, 1989, pp. 419-446.

[17] Jambunathan, R. and Levin, D. A., "Grid-Free Octree Approach for Modeling Heat Transfer to Complex Geometries," Journal of Thermophysics and Heat Transfer, Vol. 30, No. 2, 2016, pp. 379-393.

[18] Ivanov, M., Markelov, I., Taylor, S., and Watts, J., "Parallel DSMC strategies for 3D computations," Proceedings of the parallel CDS'96, 1997.

[19] Dietrich, S. and Boyd, I. D., "Scalar and parallel optimized implementation of the direct simulation Monte Carlo method," Journal of Computational Physics, Vol. 126, No. 2, 1996, pp. 328-342.

[20] Gallis, M. A., Torczynski, J. R., Plimpton, S. J., Rader, D. J., and Koehler, T., "Direct simulation Monte Carlo: The quest for speed," Proceedings of the 29th International Symposium on Rarefied Gas Dynamics, Vol. 1628, AIP Publishing, 2014, pp. 27-36.

[21] Wu, J.-S., Tseng, K.-C., and Wu, F.-Y., "Parallel three-dimensional DSMC method using mesh refinement and variable time-step scheme," Computer physics communications, Vol. 162, No. 3, 2004, pp. $166-187$.

[22] Top500.org, "Top 500, List," https://www.top500.org/lists/2016/06/, 2016, [Online; accessed 20August-2016]. 
[23] Su, C., Hsieh, C., Smith, M., Jermy, M., and Wu, J., "Parallel direct simulation Monte Carlo computation using CUDA on GPUs," Proceedings of the 27th International Symposium in Rarefied Gas Dynamics, Pacific Grove, California, USA, Vol. 1333, 2011, p. 343.

[24] Gladkov, D., Tapia, J.-J., Alberts, S., and D'Souza, R. M., "Graphics processing unit based direct simulation Monte Carlo," Simulation, 2011, pp. 0037549711418787.

[25] Zabelok, S., Arslanbekov, R., and Kolobov, V., "Adaptive kinetic-fluid solvers for heterogeneous computing architectures," Journal of Computational Physics, Vol. 303, 2015.

[26] Goldsworthy, M., "A GPU-CUDA based direct simulation Monte Carlo algorithm for real gas flows," Computers \& Fluids, Vol. 94, 2014, pp. 58-68.

[27] Tumuklu, O., Levin, D. A., and Austin, J. M., "Shock-shock interactions for a double wedge configuration in different gases," Proceedings of the 53rd AIAA Aerospace Sciences Meeting, Kissimmee, FL, 2015, pp. AIAA-2015-0580.

[28] Sawant, S. S., Jambunathan, R., Tumuklu, O., Korkut, B., and Levin, D. A., "Study of Shock-Shock Interactions Using an Unstructured AMR Octree DSMC Code," 54th AIAA Aerospace Sciences Meeting, 2016, pp. AIAA, 2016-0501.

[29] Su, C.-C., Smith, M. R., Kuo, F.-A., Wu, J.-S., Hsieh, C.-W., and Tseng, K.-C., "Large-scale simulations on multiple Graphics Processing Units (GPUs) for the direct simulation Monte Carlo method," Journal of Computational Physics, Vol. 231, 2012, pp. 7932-7958.

[30] Kashkovsky, A., Shershnev, A., and Ivanov, M., "Efficient CUDA implementation in the DSMC method," 28TH INTERNATIONAL SYMPOSIUM ON RAREFIED GAS DYNAMICS 2012, Vol. 1501, AIP Publishing, 2012, pp. 511-518.

[31] Kashkovsky, A. and Fan, J., "3D DSMC computations on a heterogeneous CPU-GPU cluster with a large number of GPUs," AIP Conference Proceedings, Vol 1628, AIP, 2014, pp. 192-198.

[32] Ericson, C., Real-time collision detection, CRC Press, 2004.

[33] Frisken, S. F. and Perry, R. N., "Simple and efficient traversal methods for quadtrees and octrees," Journal of Graphics Tools, Vol. 7, No. 3, 2002, pp. 1-11. 
[34] Burstedde, C., Wilcox, L. C., and Ghattas, O., "Scalable algorithms for parallel adaptive mesh refinement on forests of octrees," SIAM Journal on Scientific Computing, Vol. 33, No. 3, 2011, pp. 1103-1133.

[35] Panerai, F., Ferguson, J., Lachaud, J., Martin, A., Gasch, M. J., and Mansour, N. N., "Analysis of fibrous felts for flexible ablators using synchrotron hard x-ray micro-tomography," 8th European Symposium on Aerothermodynamics for Space Vehicles, 2015.

[36] Arslanbekov, R. R., Kolobov, V. I., Burt, J., and Josyula, E., "Direct simulation Monte Carlo with octree Cartesian mesh," AIAA paper, Vol. 2990, 2012.

[37] Sundar, H., Sampath, R. S., and Biros, G., "Bottom-up construction and 2: 1 balance refinement of linear octrees in parallel," SIAM Journal on Scientific Computing, Vol. 30, No. 5, 2008, pp. 2675-2708.

[38] Karypis, G., Schloegel, K., and Kumar, V., "Parmetis," Parallel graph partitioning and sparse matrix ordering library. Version, Vol. 2, 2003.

[39] Möller, T. and Trumbore, B., "Fast, minimum storage ray/triangle intersection," ACM SIGGRAPH 2005 Courses, ACM, 2005, p. 7.

[40] Bell, N. and Hoberock, J., "Thrust: a productivity-oriented library for CUDA," GPU Computing Gems: Jade Edition, 2012.

[41] Wen-Mei, W. H., GPU Computing Gems Emerald Edition, Elsevier, 2011.

[42] Ivanov, M. and Rogasinskii, S., "Theoretical analysis of traditional and modern schemes of the DSMC method," Rarefied Gas Dynamics, Vol. 1, 1991, pp. 629-642.

[43] Ivanov, M., Kashkovsky, A., Gimelshein, S., Markelov, G., Alexeenko, A., Bondar, Y. A., Zhukova, G., Nikiforov, S., and Vashenkov, P., "SMILE system for 2D/3D DSMC computations," Proceedings of 25th International Symposium on Rarefied Gas Dynamics, St. Petersburg, Russia, 2006, pp. 21-28.

[44] Hirt, C. W. and Nichols, B. D., "Volume of fluid (VOF) method for the dynamics of free boundaries," Journal of Computational Physics, Vol. 39, No. 1, 1981, pp. 201-225.

[45] Agrawal, P., Chavez-Garcia, J. F., and Pham, J., "Fracture in phenolic impregnated carbon ablator," Journal of Spacecraft and Rockets, Vol. 50, No. 4, 2013, pp. 735-741.

[46] Lachaud, J., Cozmuta, I., and Mansour, N. N., "Multiscale approach to ablation modeling of phenolic impregnated carbon ablators," Journal of Spacecraft and Rockets, Vol. 47, No. 6, 2010, pp. 910-921. 
[47] Marschall, J. and Milos, F. S., "Gas permeability of rigid fibrous refractory insulations," Journal of Thermophysics and Heat Transfer, Vol. 12, No. 4, 1998, pp. 528-535.

[48] Panerai, F., White, J. D., Cochell, T. J., Schroeder, O. M., Mansour, N. N., Wright, M. J., and Martin, A., "Experimental measurements of the permeability of fibrous carbon at high-temperature," International Journal of Heat and Mass Transfer, Vol. 101, 2016, pp. 267-273.

[49] Scheidegger, A. E., "Physics of flow through porous media," Physics of flow through porous media, No. 532.5 S2 1974, University of Toronto, 1963.

[50] Wiegmann, A., Computation of the permeability of porous materials from their microstructure by FFFStokes, Fraunhofer-Institut für Techno-und Wirtschaftsmathematik, Fraunhofer (ITWM), 2007. 